This dissertation has been $\quad 63-2546$ microfilmed exactly as received

ROBISON, Donald Edward, 1931ESTIMATES FOR THE POINTS OF INTERSECTION OF TWO POLYNOMLAL REGRESSIONS.

The Ohio State University, Ph.D., 1962 Mathematics

University Microfilms, Inc., Ann Arbor, Michigan 


\title{
ESTIMATES FOR THE POINTS OF INTERSECTION \\ OF TWO POLYNOMIAL REGRESSIONS
}

\author{
DISSERTATION
}

Presented in Partial Fulfiliment of the Requirements for the Degree Doctor of Philosophy in the Graduate School of the Ohio State University

By

Ynald Edward Robison, B.S., M.Sc.

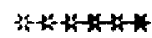

The Ohio State University 1962

Approved by

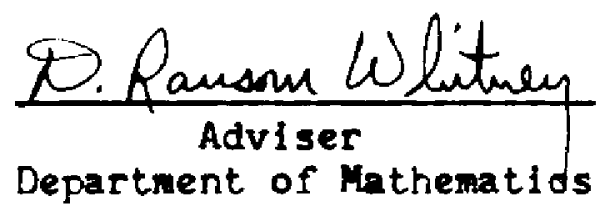




\section{ACKNONLEDGMENTS}

\section{To ny adviser,}

Professor D.R. Whitney,

who has made academic life a pleasure;

and to my family,

Enily, David, and Jeannie,

who make the rest of 1 ife a pleasure. 


\section{CONTENTS}

Chapter

I Introduction

1.1 Description of Problem 1

1.2 A Graphical Example 2

1.3 Definitions and Notation 3

1.4 Maximum Likelihood Theory of Estimation 5

1.5 MuItiple Regression Theory

1.6 Summary of the Results in this Paper

II Simple Polynomial Regression: Discrete Model 11

2.1 Statement of the Problem 11

2.2 Intersecting Polynomials, $N_{1}$ and $N_{2}$ Known 12

2.2.1 The Maximu Likelihood Estimators for $\left(t_{*}, x_{*}\right)$

2.2 .2 Computational Fornulas for $t_{*}$ and $x_{*}$.

2.2.3 Confidence Intervals for $t_{*}$ * 20

2.3 Explicit Results

2.3.1 Polynomials of lst Degree 25

2.3.2 Polynomials of 2nd Degree 33

2.3.3 Polynomials of $k$-th Degree 38

2.4 Discussion of Practical Difficulties 39

2.4.1 Examination of the Assumptions and the Sample 39

2.4.2 More Than One Root in I 42

2.4.3 Real Solutions, But None in I 42

2.4.4 No Real Solutions in I 42

2.4.5 The Method of Intersecting Confidence Regions 49

2.5 A Jump at the Intersection 50

$2.6 \quad N_{1}$ and $N_{2}$ Not known 52

2.6.1 No Regression Assumptions 53

2.6.2 With Regression Assumptions: Comments 70

III Simple Linear Regression: Continuous Model 76

3.1 Definition of the Problem 76

3.2 Fundamental Random Process 77

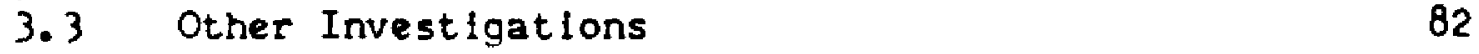

$\begin{array}{ll}\text { Bibliography } & 85\end{array}$

$\begin{array}{ll}\text { Autobiography } & 87\end{array}$ 


\section{ESTIMATES FCR THE POINTS OF INTERSECTION \\ OF THO POLYNOMIAL REGRESSIONS}

\section{CHAPTER I}

INTRODUCTION

\subsection{Description of the Problem}

Research workers in the experimental sciences are frequently confronted with experimental data in which a sudden and decisive change in trend is observed. It is of interest to know where or when this change takes place. The topic of this paper is the statistical estimation of these points of change in trend.

The first point of view is to regard the data as samples from two intersecting normal processes. The samples taken are assumed to be discrete but not necessarily evenly spaced with respect to any parameter. The problem at hand is to provide estimates and distribution theory for the point or points of intersection of the two estimated regression curve. This problem will be studied under various polynomial assumptions for the form of the regression curves. The solution obtained consists of providing maximus likelihood estimates and confidence regions when such exist, and of presenting alternative procedures otherwise.

A more general point of view is to regard the data as samples from two intersecting processes for which continuous sample records are available. The probler is to provide estifites and their distributions 
for the points of intersection of the regressions of the processes. This problem will be studied for the case of simple linear regression from a Fundamental Fandom Process. Estimates of the intersection and corresponding confidence regions are obtained.

\subsection{A Graphical Example}

There are a variety of situations where tre point of intersection, $P$, is of interest. The poirt $P$ migit corresfond to some maximum or minimur of some physical frocess. The abscissa of P right correspond to a position of demarhution, to the left of which interpolation and extrapolation would be pericumed according to a first function, and to the right of which the same operations would be performed by a second function. In the case of missing interior data points, P right be used to establish a thread of continuity throust the missing region. Finally, $P$ may be of just plain academic interest.

As an explicit example consider a sample taken from telemetry data from an upper atmospheric research proslem. Radiation belts are known to exist above the earth, but the radial distances of certain intensities varies from time to time. Suprose the experiment provides intensities and times as coordinate pairs. Sudien changes in trend of radiation as a function of time are krow to occur. Knowledge of the exact time of this change may be of considerable interest (Figure 1). By visual inspection the point of change seems to be somewhere near $t$ * It is desired to have a sound statistical estimate for the abscissa of this point. 


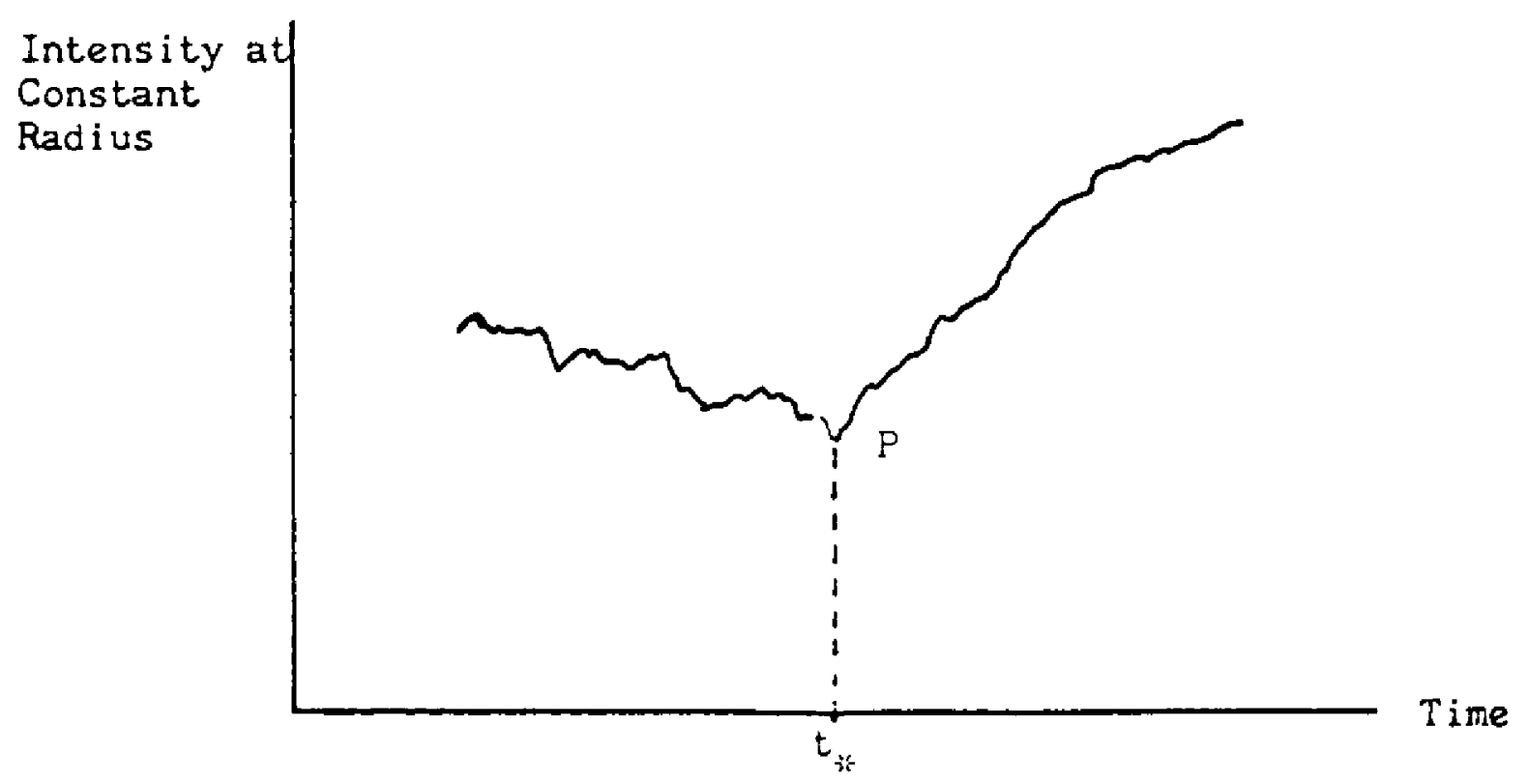

Figure 1

1.3 Definitions and Notation

Usually we shall try to denote random variables by carital letters and fixed observations of $r$ andom variables by lower case letters. However, we shall not forsake the convenience of denoting maximum likelihood estimates of a number $a$ by $\hat{a}$ and other estimates of $a$ by $\tilde{\mathbf{a}}$ :

A stochastic process is an indexed set of randor variables, $\left\{x_{t}\right\}$. For our purposes $t$ will be selected from some set of real numbers. It is convenient to ragard $t$ as time. For any particular value of $t$, say $t_{0}$, the symbcl $x_{t_{0}}$ is a random variable. A frocess is considered known if for any finite set $\left(t_{1}, \ldots, t_{n}\right)$ the joint distribution of $\left(x_{t_{1}}, \ldots, x_{t_{n}}\right)$ is known. When there is little chance of misinterpretation we shall shorten the notation $\{x\}$ to $x_{t}$. 
A sample function from a process $x_{t}$ is a function $x(t)$ composed of a single observation of $X_{t}$ for each $t$ in some subset of t's. A sample function can be either discrete or continuous.

The mean value function for a process is the function $m(t)$ which gives the expectation $i x_{t}$ at any time $t$. It is usually called the regression function of $x_{t}$. The covariance function is the function $\sigma_{12}=\sum\left[x_{t_{1}}-\pi_{1}\left(t_{1}\right)\right]\left[x_{t_{2}}-m\left(t_{2}\right)\right]$. The variance function is obtained from the covariance function when $t_{1}=t_{2}=t$. It is denoted $\sigma_{t}^{2}$.

Consider any function $g(t)$ associated with a process $x_{t}$ and consider also the corresponding function $g$ ir(t) associated with a second process $\mathrm{X}_{t}{ }^{*}$. The two processes are said to intersect: if the associated functions $g$ and $g^{*}$ intersect in the usual geometric sense. In this paper we consider only processes whose regression functions intersect. This definition stall include the special case when a process $x_{t}, t \varepsilon T$, is considered as the union of two other processes $X_{t}^{\prime}, t^{\prime} \varepsilon T^{\prime}$, and $X_{t}^{\prime \prime}, t^{\prime \prime} \varepsilon T^{\prime \prime}$, with $T^{\prime}+T^{\prime \prime}=T$, so that the associated functions $g^{\prime}$ ard $g^{\prime \prime}$ can be regarded as intersecting branches of a more inclusive $g(t)$.

Most of this paper will be concerned with discrete samples from two processes $X_{t}$ and $Y_{t}$. But in Chapter III one continuous model will be studied, requiring the following definition (Mann, 1953). A Fundamental Random Process (FRP) is a process $x_{t}$ satisfying 1) $x_{t+\tau}-x_{t}=\varepsilon_{t, \tau}$ where $\varepsilon_{t, \tau}$ is a random variable with zero mean and is indepencient of $x_{t}$ and also of $\varepsilon_{t \prime}, T^{\prime}$ if the 
intervals $(t, t+\tau)$ and $\left(t^{\prime}, t^{\prime}+\tau\right)$ do not overlap.

2) The distribution of $\varepsilon_{t, \tau}$ depends only on $\tau$.

3) The variance of $\varepsilon_{t, \tau}$ exists and is given by Here, $c$ is a positive constant.

4) Large values of $\varepsilon_{t, \tau}$ are uniikely; precisely, it is required that for sufficiantly small $\tau$ and arbitrary $\rho>0$ and $\eta>0, \int_{|a|>\rho} \rho^{2} d F_{\tau}(a)<\eta \sigma^{2}$, where $F_{\tau}(a)$ is the distribution function of $\epsilon_{t, \tau}$.

Thus an FRP has a single parameter, c. As a consequence of the definition it can be shown that $\varepsilon_{t, \tau}$ is normally distributed with variance $c$.

We will often consider a related process $Y_{t}=f(t)+X_{t}$, where $f(t)$ is a non-random regression function, usually unknown, and where $x_{t}$ is an FRP. Another related process is $z_{t}=\int_{0}^{t} x_{s} d s$. If $x_{s}$ is an FRP then $z_{t}$ is Gaussian with mean zero and covariance

$$
\sigma_{t, t^{\prime}}=\frac{c}{2} \max \left(t, t^{\prime}\right)\left[\min \left(t, t^{\prime}\right)\right]^{2}-\frac{c}{6}\left[\min \left(t, t^{\prime}\right)\right]^{3} \text {. }
$$

\subsection{Maximum Likelihood Theory of Estimation}

The principle of maximum likelihood (M.L.) states that to estimate parameters $\left(\theta_{1}, \ldots, \theta_{k}\right)$ from a sample $\left(x_{1}, \ldots, x_{n}\right)$ one should form the joint probability function of the sample and then find the values $\left(\hat{\theta}_{1}, \ldots, \hat{\theta}_{k}\right)$ that maximize this function. This function is called the likelihood function.

In this paper we shal I use the likelihood priaciple not only in the 
above context but also in the case when the data are given by a continuous sample record. To proceed, suppose $y(t)$ is given on $[0, T]$. Form a subdivision of $(n+1)$ points from $[0, T]$ and consider the discrete sample points $\left(y_{0}, y_{1}, \ldots, y_{n}\right)$. The likelihood function for this sample can be formed and a solution $\hat{\theta}$ obtained. Now consider a sequence of such subdivisions as the modulus converges to zero. If $\hat{\theta}$ converges in some probability sense to a limiting estimator, we shall refer to that limiting random variable as the maximum likelihood estimate in the continuous case.

Mann(1954) has siown that for the multiple regression estimation problem assoclated with the FRP the limiting estimators exist as limits in the mean and are uniased.

\subsection{Multiple Regression Theory}

In this section we review the standard results in multiple regression theory. Let $Y$ denote an observable random variable and let $g_{1}, \ldots, g_{k}$ denote $k$ coservable nonrandom parameters. Consider a sample consisting of $n$ independent sets $\left(y_{i}, g_{1 i}, \ldots, g_{k i}\right)$, where $g_{j i}$ represents the $i-t h$ observation on the $j$-th parameter, $i=1, \ldots, n$. We assume

$$
y_{i}-g_{1 i} \beta_{1}+\ldots+g_{k i} \beta_{k}+\varepsilon_{i}, \quad k \leq n
$$

where the $\varepsilon_{i}$ are independent normal variates with zero means and constant variances $\sigma^{2}$; the $\beta_{j}$ are constants to be estimated. It is necessary that the rank $\left(g_{j i}\right)=k$. 
7

The solution for the maximum likelihood estimates $b_{p}$ of the $\beta_{p}$ and $\hat{\sigma}^{2}$ of $\sigma^{2}$ is well known, (Mood, p. 301 ). Let

$$
\alpha_{p q}=\sum_{i=1}^{n} g_{p i} g_{q i} \text { and } c_{q}=\sum_{i=1}^{n} y_{i} g_{q i}
$$

Then the normal equations are

$$
\sum_{p=1}^{k} a_{p q}^{b}=c_{q} \quad q-1, \ldots, k
$$

and the solution is

$$
b_{p}=\sum_{q=1}^{k} a_{q}^{p q_{c}} \text {, where }\left(a^{p q}\right)-\left(a_{p q}\right)^{-1}
$$

Furthermore

$$
\hat{\sigma}^{2}=\frac{1}{n} \sum_{i=1}^{n}\left(y_{i}-g_{1 i} b_{1}-\cdots-g_{k i} b_{k}\right)^{2}
$$

The $b_{p}$ are unbiased estimates of the $\beta_{p}$. They are jointly normally distributed and the elements of the covariance matrix are given by

$$
\sigma_{b_{p} b_{q}}=a^{p q \sigma^{2}} \quad, \quad p, q-1, \ldots, k
$$

It can be shown that $n \hat{\sigma}^{2} / \sigma^{2}$ has the chi square distribution $(n-k)$ degrees of freedom and that it is independently distributed of the $b_{p}$. To obtain polynomial regressions in time, take $g_{i-1}=t^{1}$. 
1.6 A Sumary of the Results in This Paper

Chapter II deals with the intersections of two polynomial regressions in the context of a discrete sampling procedure. It is assumed that a first process $X_{t}$ precedes a second process $Y_{t}$ in time. Points $P$ of intersection are denoted by $\left(t_{*}, x_{\sharp}\right)$. Usually it can be assumed that some true $t_{*}$ falls in a known interval I corresponding to the time lag after the first process and preceding the second. If the sample consists of values $\left(x_{1}, \ldots, x_{N_{1}}, y_{1}, \ldots, y_{N_{2}}\right)$ at times $\left(s_{1}, \ldots, s_{N_{1}}, t_{1}, \ldots, t_{N_{2}}\right)$ then $I$ is the interval $\left[s_{N_{1}}, t_{1}\right]$. The maximum likelihood estimates of $P$ are obtained and are found to be the intersections of the sample M.L. regressions. But the M.L. estimates of $P$ do not always exist because the two sample polynomials may not always intersect. The M.L. formulation is checked for correctness and the results are affirmative. Thus If practical difficulties arise, it is the falit of the sample and not of the general theory. For cases when the M.L. estimates do exist in $I$, confidence intervals are obtiined for $t_{\star}$. For cases where the M.L. estimators exist and lie outside I (but correspond to some $t_{k}$ in $I$ ) a revised point estimator is found to be the endpoint of $I$ nearest $\tilde{t}_{\#}$. Confldence linits for $t_{*}$ are again found and the intersection of these with $I$ is taken as an improved confidence interval for $t_{*}$, thus completing the distribution theory. Explicit examples for polynomials of degree one and two are presented.

For cases where the M.L. estimates clearly do not correspond 
to a $t_{*}$ in $I$, or where the sample regressions completely fail to intersect, three alternative procedures are considered. The preferred metiod is to find an optimal point $\left(\tilde{t}_{*}, \hat{x}_{\#}\right)$ which could have been included in the regression estimates and which would have minimized an associated likelihood-type function. The solution for such a point is numerically difficult because of the functional interdependence of all of the parameters. Another method is that of finding the abscissa in I where the sample regressions are closest. This method is easy, but has certain shortcomings. A third method is that of constructing confidence bands about each of the sample regressions and to consider the intersection of these bands in $I$ as defining a confidence region for $P$. This method is not always possible. The common virtue of all three methods is that they all recover the N.L. estimate when $\tilde{t}_{*}$ is in $I$. In many cases the interval I may not be known exactly. In such cases the likelitood is also a function of $\mathrm{N}_{1}$. The correct procedure is to try various divisions of the sample, corresponding to various choices of $N_{1}$ and $N_{2}$. For each such choice calculate separate regression estimates and pick the choice which eventually maximizes the likelihood.

With I not known exactly, probabilities associated with selecting $\left(\mathrm{N}_{1}, \mathrm{~N}_{2}\right)$ are of interest. These probabilities are derived for the non regression case under conditions of both correct and incorrect selection. These results are of interest in the classical two-sample problem. When regression assumptions are added, the selection probabilities are difficult to calculate. 
Some progress on this problem, in terms of approximations, is reported.

Chapter III deals with simple 1 inear regressions for two fundamental randon processes. Under a special assumption on the F.R.P. the distribution of $t_{*}$ is obtalned. 
CHAPTER II

THE INTERSECTION OF SIMPLE POLYNOMLAL REGRESSIONS:

DISCRETE MODEL

\subsection{Statement of the Problex}

In a simple regrassion problem the regression is assuned to be function of Just one observable parameter, say $t$. Polynomial regressions are polynomials in the variable $t$. The paraneter $t$ will be called time.

Consider a process with unknown regression $\mu(t)$ having two intersecting branches $X_{s}$ and $Y_{t}$ such that

$$
\mu(\cdot)= \begin{cases}E\left(X_{s}\right)=\mu_{1}(s)=+a_{1} s+\ldots+a_{k} s^{k} & \text { if } s \leqq t \\ E\left(Y_{t}\right)=\mu_{2}(t)=\beta_{0} i_{1} t+\ldots+\beta_{q} t^{q} & \text { if } t \geqq t_{*}\end{cases}
$$

The variables $s$ and $t$ are neasured on the same axis and in the same units; different letters have been used simply for easy identification with $X$ and $Y$. Let the variances, $\sigma_{1}^{2}$ and $\sigma_{2}^{2}$. of $X_{s}$ and $Y_{t}$ be constant. For the discrete case no covariance structure is required. The point $t_{*}$ is an unknown abscissa of intersection of the two branch regressions. The corresponding ordinate of intersection w111 be denoted by $x_{*}$. We asssume that $x_{s}$ precedes $Y_{t}$ and that each is undefined in the other's domain. Thus we shall not consider the problea of intersecting regressions if $X_{s}$ and $Y_{t}$ overlap. Initially we shall assume that $\mu(t)$ is continuous at $t_{\text {* }}$. 
For the case of jump at the intersection see the coments in section 2.5 . Assume $k \geq q$; if this is not the case interchange the roles of $k$ and $q$.

Given a discrete sanple from each process, our problem is to estimate $t_{\text {* }}$ and to determine the distribution of the estinator. This problem will be studied under the usual independence and normality assumptions. This means that discrete samples from $X_{s}$ and $Y_{t}$ at times $s_{i}, t_{j}$ will be regarded as observations on independent normal random variables $X_{i}-X_{s_{1}}$ and $Y_{j}=Y_{t_{j}}$ having means $\mu_{1}\left(s_{1}\right)$ and $\mu_{2}\left(t_{j}\right)$ and constant variances $\sigma_{1}^{2 J}$ and $\sigma_{2}^{2}$.

Our solution, when possible, consists of presenting the maximum 11kelihood point estiator for $t$ and of showing a wethod for constructing confidence intervals about $t_{*}$. These results rely heavily on being able to solve certain sample polynomials for all real roots. When $a 11$ roots are not real, certain alternative procedures are suggested.

2.2 Intersecting Polynomials of Arbitrary Degree: $y_{1}$ and $y_{2}$ Known

Let $\left(x_{1}, \ldots, x_{y_{1}}, y_{1}, \ldots, y_{y_{2}}\right)$ be a glven set of independent observations on $X_{s}$ and $Y_{t}$ at given ordered times $\left(s_{1}, \ldots, s_{N_{1}}\right.$, $\left.t_{1}, \ldots, t_{N_{2}}\right)$. Let $N=N_{1}+w_{2}$. Then the 1ikelihood for this composite semple is a function of $(k+1)+(q+1)+2$ continuous paraneters and two discrete parameters: 


$$
\begin{aligned}
& L\left(a_{0}, \ldots, a_{k}, \beta_{0}, \ldots, \beta_{q}, \sigma_{1}^{2}, \sigma_{2}^{2} ; N_{1}, N_{2}\right) \\
& \prod_{i=1}^{N} \frac{1}{\sqrt{2 \pi} \sigma_{1}} e^{-\frac{1}{2 \sigma_{1}} 2\left[x_{1}-\mu_{1}\left(s_{i}\right)\right]^{2}} \cdot \prod_{i=1}^{N_{2}} \frac{1}{\sqrt{2 \pi} \sigma_{2}} e^{-\frac{1}{2 \sigma_{2}} 2\left[y_{i}-\mu_{2}\left(t_{1}\right)\right]^{2}}
\end{aligned}
$$

The $\alpha_{1}, \beta_{j}$ and $\sigma^{2}$ 's are unknown parameters which will be estimated by naximizing $\log L$. For the remainder of this section regard $N_{1}$ and $H_{2}$ as known. See section 2.6 for the case when $H_{1}$ and $w_{2}$ are unknown. This formulation of the likelihood equation will later be referred to as the "original" formulation.

The required $t_{*}$ is defined by equating $\mu_{1}$ and $\mu_{2}$ in the intervel $I:\left[s_{\mathrm{H}_{1}}, t_{1}\right]$,

$$
a_{0}+a_{1} t_{*}+\ldots+a_{k} t_{*}^{k}=\beta_{0}+\beta_{1} t_{*}+\ldots+\beta_{q *} t^{q} .
$$

Let

$$
\gamma_{j}= \begin{cases}a_{j}-\beta_{j} & \text { for } j=0,1, \ldots, q \\ a_{j} & \text { for } j>q .\end{cases}
$$

Then $t_{*}$ is any solution to the system

$$
\gamma_{0}+\gamma_{1} t_{*}+\ldots+\gamma_{k} t^{k}=0, \quad s_{N_{1}} \leq t_{*} \leq t_{1} .
$$

We would like to assune that $t_{*}$ is unique in I since that is the problem under consideration. But since polynomial regressions may be only approximations to the true regression, and since in nyses I may be large relative to the slze of the sampling intervals, it is more prudent to study all $k$ abscissae defined by the equation 


$$
\mu_{1}\left(t_{*}\right)-\mu_{2}\left(t_{*}\right)=0
$$

After finding a set of solutions for $t^{*}$ we would then pick that $t_{*}$ which falls in $I$. If there are more than one such $t_{\text {\# which falls }}$ in I we must establish sone decision procedure. If no $t_{*}$ falls in I we must establish alternative procedures.

\subsubsection{The Maximum Likelihood Estinates for ( $t_{*}, x_{*}$ )}

Since $t_{\#}$ is an unknown quantity which is to be estiated one might wonder why it does not occur explicitly in the likelihood function. The answer is that it could, but that the situation is easier if it doesn't. This conclusion follows from the following discussion. We will demonstrate that from the likelihood standpoint all Information about $t_{*}$ is obtainable from estimates of the a's and $\beta$ 's.

Fron the likelihood function consider the set of independent parameters $\left(a_{0}, a_{1}, \ldots, a_{k}, \beta_{0}, \ldots, \beta_{q}\right)$. The equations defining $\gamma_{j}$ define a $1-1$ transformation from parameters $\left(\alpha_{0}, \ldots, \beta_{q}\right)$ into parameters $\left(\gamma_{0}, \gamma_{1}, \ldots, \gamma_{k}, \beta_{0}, \ldots, \beta_{q}\right)$. If $t_{*}^{(1)}, \ldots, t_{*}^{(k)}$ denote the $k$ solutions $f \times t_{*}$ then

$$
\begin{aligned}
\gamma_{0}+\gamma_{1} t_{*}+\ldots+\gamma_{k} t_{*}^{k} & =\gamma_{k}\left(\delta_{0}+\delta_{1} t_{*}+\ldots+\delta_{k-1} t^{k-1}+t_{*}^{k}\right) \\
& =\gamma_{k}\left(t_{*}-t_{*}^{(1)}\right) \cdot \ldots \cdot\left(t_{*}-t_{*}^{(k)}\right)
\end{aligned}
$$

where $\delta_{j}=\gamma_{j} / \gamma_{k}, j=0,1, \ldots, k-1$.

But from the theory of equations the $\delta_{j}$ are expressible in 1-1 
fashion with the $t(j)$ :

$$
\begin{aligned}
\delta_{k-1} & =-\left(\text { sum of the roots, } t_{*}^{(j)}\right) \\
\delta_{k-2} & =+(\text { sum of the products of the roots taken } 2 \text { at a time) } \\
& : \\
\delta_{0} & :(-1)^{k} \cdot\left(\text { product of all the roots, } t_{*}^{(j)}\right)
\end{aligned}
$$

Thus there exists a 1-1 transformation from the parameter set $\left(\alpha_{0}, \ldots, \beta_{q}\right)$ into the parameter set $\left(t_{*}^{(1)}, \ldots, t_{*}^{(k)}, \gamma_{k}, \beta_{0}, \ldots, \beta_{q}\right)$. Consider now the following theoren given by Anderson ( $p .48)$.

Theorem. If on the basis of a given sample, $\hat{\theta}_{1}, \ldots, \hat{\theta}_{\mathrm{m}}$ are $\operatorname{maximum}$ Iikelihood estimates of parameters $\theta_{1}, \ldots, \theta_{m}$ of a distribution, then $\hat{\theta}_{1}\left(\hat{\theta}_{1}, \ldots, \hat{\theta}_{m}\right), \ldots, \hat{\theta}_{m}\left(\hat{\theta}_{1}, \ldots, \hat{\theta}_{m}\right)$ are maximum 11 kelihood estimates of $\phi_{1}\left(\theta_{1}, \ldots, \theta_{m}\right), \ldots, \phi_{m}\left(\theta_{1}, \ldots, \theta_{m}\right)$ if the transformation from $\theta_{1}, \ldots, \theta_{\mathrm{m}}$ to $\phi_{1}, \ldots, \phi_{\mathrm{m}}$ is one-to-one. If the estimates of $\theta_{1}, \ldots, \theta_{m}$ are unique then the estimates of $\emptyset_{1}, \ldots, \phi_{m}$ are unique. Applying this result to the $1-1$ correspondence demonstrated above, it is evident that the M.L. estimators of the $t_{*}$ are obtainable from the M.L. estimates of the a's and $\beta^{\prime}$. We shall soon see that this explicitly involves solving the polynomial equation obtained from equating the sample regressions $\hat{\mu}_{1}$ and $\hat{\mu}_{2}$. Thus it is not necessary that the $t_{\#}$ occur explicitiy in the likellhood function since $\left(\alpha_{0}, \ldots, \beta_{q}\right)$ and $\left(t_{*}^{(1)}, \ldots, \beta_{q}\right)$ are equivalent parameter sets. The choice is made in favor of $\left(\alpha_{0}, \ldots, \beta_{q}\right)$ because the other choice produces likelihood equations which are more difficult 
to solve.

It would seen that the existence of the M.L. estimators depends on the existence of real solutions in I of the polynowial $\hat{\mu}_{1}\left(t_{*}\right)=\hat{\psi}_{2}\left(t_{*}\right)$. This observation prompted the author to consider alternative formulations of the likelihood equation, such as in terms of the parameter set just discussed, to see if there was a more ravorable likelihood formulation. The answer is, of course, in the negative, but two of these formulations will be discussed in passing.

One possiblity consicered was that of maximizing $L$ subject to the "condition" that $\mu_{1}\left(t_{k}\right)=\mu_{2}\left(t_{k}\right)$ by using Lagrange multipliers. But further study indicated that this was not truly a restriction on ( $\left.\alpha_{0}, \ldots, \beta\right)$ but rather an equation defining alternative parameters $\left(t^{(1)}, \ldots, t^{(k)}\right)$. These could replace any $k$ of the $\alpha^{\prime} s$ or $\beta^{\prime} s$. Another possibllity studied was to write each regression as a polynomial about some root, notationally designated as $t_{*}^{(1)}$, which lies in $I$ :

$$
\begin{aligned}
& \mu_{1}(t)=\alpha_{0}^{*}+\alpha_{1}^{*}\left(t-t_{*}^{(1)}\right)+\ldots+\alpha_{k}^{*}\left(t-t_{*}^{(1)}\right)^{k} \\
& \mu_{2}(t)=\beta_{0}^{*}+\beta_{1}^{*}\left(t-t_{*}^{(1)}\right)+\ldots+\beta_{q}^{*}\left(t-t_{*}^{(1)}\right)^{q} .
\end{aligned}
$$

Then the $t_{*}^{(j)}$ are solutions to

$$
\begin{aligned}
P(t)=\left(\alpha_{0}^{*}-\beta_{0}^{*}\right) & +\left(\alpha_{1}^{*}-\beta_{1}^{*}\right)\left(t-t_{*}^{(1)}\right)+\ldots \\
& +\left(a_{k}^{*}-\beta_{k}^{*}\right)\left(t-t_{*}^{(1)}\right)^{k}=0, \beta_{j}^{*} \text { o for } j>q_{.}
\end{aligned}
$$

In particular, $P\left(t_{*}^{(1)}\right)=0$ means that $\sigma_{0}^{*}=\beta_{0}^{*}$. Hence $\left(t_{*}^{(1)}, \alpha_{1}^{*}, \ldots, \beta_{q}^{*}\right)$ is an alternative parameter set. Furthermore, 
knowledge of this set and of the identities

$$
\begin{aligned}
& a_{0}+a_{1} t+\ldots+a_{k} t^{k}=\alpha_{0}^{*}+a_{1}^{*}\left(t-t_{*}^{(1)}+\ldots+\alpha_{k}^{*}\left(t-t_{*}^{(1)}\right)^{k}\right. \\
& \left.\beta_{0}+\beta_{1} t+\ldots+\beta_{q} t^{q}=\beta_{0}^{*}+\beta_{1}^{*}\left(t-t_{*}^{(1)}\right)+\ldots+\beta_{q}^{*}(t-t)^{(1)}\right)^{q}
\end{aligned}
$$

will recover $\left(\alpha_{0}, \ldots, \beta_{q}\right)$ explicitly. Hence there exists a $1-1$ transformation between $\left(\alpha_{0}, \ldots, \beta_{q}\right)$ and $\left(t_{*}^{(1)}, \alpha_{1}^{*}, \ldots, \beta_{q}^{*}\right)$. Since these parameter sets are equivalent we select $\left(\alpha_{0}, \ldots, \beta_{q}\right)$ because of easler likelihood equations.

Thus it is argued that the original likelihood formulation given is correct and is the easiest such formulation to work with. This conclusion is of great importance when we see that the M.L. estimator of $t_{*}$ doesn't always exist. We immediately wonder if our likelihood formulation is correct, and the previous argument shows that it is .

We now turn to the mechanics of imclementing the preceding solution. We will obtain the M.L. estimator and then show a way of deriving confidence intervals for the $t_{*}$.

2.2.2 Computation Formulas for $t_{*}$ and $x_{*}$

Taking logarithms of the likelihood function we obtain

$$
\begin{aligned}
\log L= & -\frac{N}{2} \log 2 \pi-\frac{N_{1}}{2} \log \sigma_{1}^{2}-\frac{N_{2}}{2}-\log \sigma_{2}^{2} \\
& -\frac{1}{2 \sigma_{1}^{2}} \sum_{i=1}^{N_{1}}\left[x_{i}-\mu_{1}\left(s_{i}\right)\right]^{2}-\frac{1}{2 \sigma_{2}^{2}} \sum_{i=1}^{N_{2}}\left[y_{i}-\mu_{2}\left(t_{i}\right)\right]^{2} .
\end{aligned}
$$


By maximizing $\log L$ we can separately obtain the usual (section 1.6) maximum likelihood estimates $a_{i}$ of $a_{i}$ and $b_{j}$ of $b_{j}$. The estimates for the regressions and variances are:

$$
\begin{aligned}
& \hat{\mu}_{1}=m_{1}(s)=a_{0}+a_{1} s+\ldots+a_{k} s^{k} \quad \text { (based on } x_{1}, \ldots, x_{N_{1}} \text { ) } \\
& \hat{H}_{2}=w_{2}(t)=b_{0}+b_{1} t+\ldots+b_{q} t^{q} \quad \text { (based on } y_{1}, \ldots, y_{N_{2}} \text { ) } \\
& \hat{\sigma}_{1}^{2}=\frac{1}{N_{1}} \sum_{i=1}^{N_{1}}\left[x_{i}-m_{1}\left(s_{i}\right)\right]^{2} \\
& \hat{\sigma}_{2}^{2}=\frac{1}{N_{2}} \sum_{i=1}^{N_{2}}\left[y_{i}-m_{2}\left(t_{i}\right)\right]^{2} .
\end{aligned}
$$

It is krown that the $\left(a_{0}, \ldots, a_{r}\right)$ are jointly normally distributed with means $\left(a_{0}, \ldots, a_{r}\right)$ and covariances $\sigma_{a_{1}, j}=r^{i j} \sigma_{1},(i, j-1$ to $k)$, where each $r^{1\}}$ is a function of $\left(s_{1}, \ldots, s_{N_{1}}\right\}^{3}$ only and is to be identified with $a^{1 \mathrm{~J}}$ in section 1.6 . Likewise the $\left(b_{0}, \ldots, b_{s}\right)$ are jointly norma $11 y$ distributed with means $\left(\beta_{0}, \ldots, \beta_{s}\right)$ and covariances $\sigma_{b_{1} b_{j}}=s^{1 j} \sigma_{2}^{2}(i, j-1, \ldots, s)$, where each $s^{i j}$ is a function of $\left(t_{1}, \ldots, t_{N_{2}}\right)$ only and is also to be identified with $a^{i j}$ in section 1.6 . The $\left(a_{0}, \ldots, a_{r}\right)$ and $\left(b_{0}, \ldots, b_{s}\right)$ are distributed independently of themselves and of $\hat{o}_{1}^{2}$ and $\hat{o}_{2}^{2}$.

Equating the sample regressions $m_{1}(t)$ and $m_{2}(t)$ we obtain

$$
a_{0}+a_{1} \hat{t}_{*}+\ldots+a_{k} \hat{t}^{k}=b_{0}+b_{1} \hat{t}_{*}+\ldots+b_{q} \hat{t}^{q}
$$


Let

$$
c_{j}= \begin{cases}a_{j}-b_{j} & \text { for } j=0, \ldots, q \\ a_{j} & \text { for } j>q\end{cases}
$$

We note that the $c_{j}$ are jointly normal and are unbiased estimates of the $\gamma_{j}$. Then the required estimates $\hat{t}_{*}$ are solutions to the system

$$
\begin{aligned}
& c_{0}+c_{1} \hat{t}_{*}+\ldots+c_{k} \hat{t}^{k}=0 \\
& s_{v_{1}} \leqq \hat{t}_{*} \leqq t_{1}
\end{aligned}
$$

Furthermore $\hat{x}_{*}-\hat{\mu}_{1}\left(\hat{t}_{*}\right)$ for any $\hat{t}_{*}$ in $I$. The above polynomial, with coefficients $c_{j}$, will be called the $t_{*}$-estimator polynomial.

What should our M.L. estimator be if the sample regressions don't Intersect? The answer, in view of the correctness of the likelihood formulation argued proviously, is that the M.L. estimators simply don't exist. The maximization situation is completely determined. There are no free parameters to adjust (such as $t_{*}$ ) to determine other smooth maxima. There is nc polnt to looking for a maximan other than by the use of partial derivatives since $L$, being quadratic in the $\alpha^{\prime}$ 's and $\beta$ 's, has only smooth extrema. The estimates of $a^{\prime}$ s and $\beta^{\prime}$ 's contain all likelihood information about $t_{\#}$ * We still be able to find al ternative estimators, but they won't be M.L. estimators. For fixed $(k, q)$ and a fixed sample, we have gone as far as we can go with M.L. estimation theory. We conclude with the following theorem, which summarizes the discussions in the last several pages. 
Theoren. Let $\hat{\mu}_{1}(t)$ and $\hat{\mu}_{2}(t)$ be maximum likelihood estimates of true polynomial regressions $\mu_{1}(t)$ and $\mu_{2}(t)$. Let the abscissae of intersection be defined by equating $\mu_{1}\left(t_{*}\right)=\mu_{2}\left(t_{*}\right)$. If the maximum likelihood estimators of $t_{*}$ exist as real numbers, they are given by the roots of the sample equation $\hat{\mu}_{1}\left(\hat{t}_{*}\right)=\hat{\mu}_{2}\left(\hat{t}_{*}\right)$. The estimate of the ordinates, $\hat{x}_{*}$, of intersection are the commen values of $\hat{\mu}_{1}\left(\hat{t}_{*}\right)$ and $\hat{\mu}_{2}\left(\hat{t}_{*}\right)$ for any real $\hat{t}_{*}$.

\subsubsection{Confidence Intervals for $t_{*}$}

We would like to find the distribution of $\hat{t}_{*}$. But since $\hat{t}_{*}$ is a solution to a polynomial equation there is In general no explicit formula for any $\hat{t}_{\#}$ in terms of the equations's coefficients. This and other situations make the distribution problem virtually unsolvable. He surmount this difficulty by seeking confidence intervals for $t_{*}$ directly. The intervals obtained contain $\hat{t}_{*}$ but are not necessarlly symetric about it.

Consider the random variable

$$
u=c_{0}+c_{1} t_{*}+\cdots+c_{k} t^{k}
$$

This random variable is normally distributed because 1 is is a 1 inear combination of normal variates. Note that $u$ contains the true $t_{*}$ and not the M.L. estimator. The mean of $u$ is zero since

$$
E[u]=\gamma_{0}+\gamma_{1} t_{*}+\ldots+\gamma_{k} t_{*}^{k}=0
$$

The variance of $u$ is given by 
21

$$
\sigma_{u}^{2}=\sum_{i=0}^{k} \sum_{j=0}^{q} t^{i+j} \sigma_{c_{i} c_{j}}
$$

But

$$
\begin{aligned}
& \sigma_{c_{i} c_{j}}= \begin{cases}\sigma_{\left(a_{i}-b_{i}\right)\left(a_{j}-b_{j}\right)} & 1=1, \ldots, q \quad j=1, \ldots, q \\
\sigma_{a_{1} a_{j}} & 1=1, \ldots, k \quad j=q+1, \ldots, k\end{cases} \\
& =\left\{\begin{array}{l}
\sigma_{1} a_{j}+\sigma_{b_{i}} b_{j} \\
\sigma_{a_{i}} a_{j}
\end{array}\right. \\
& =\left\{\begin{array}{l}
r^{i j_{\sigma_{1}}^{2}}+s^{1 j_{\sigma_{2}}^{2}} \\
r^{i j_{\sigma_{1}}^{2}}
\end{array}\right.
\end{aligned}
$$

where the $r^{1 j}$ and $s^{1 j}$ are the elements of the inverses of the matrices of coefficients obtained from the s's and t's.

Thus

$$
\begin{aligned}
& \sigma^{2}=\left(\sum_{i=0}^{q}+\sum_{i=q+1}^{k}\right)\left(\sum_{j=0}^{q}+\sum_{j=q+1}^{k}\right) t^{i+j} \sigma_{c_{1} c_{j}} \\
& =\sum_{i=0}^{q} \sum_{j=0}^{q} t^{i+j}\left(r^{1 j} \sigma_{1}^{2}+s^{1 j \sigma_{2}^{2}}\right)
\end{aligned}
$$

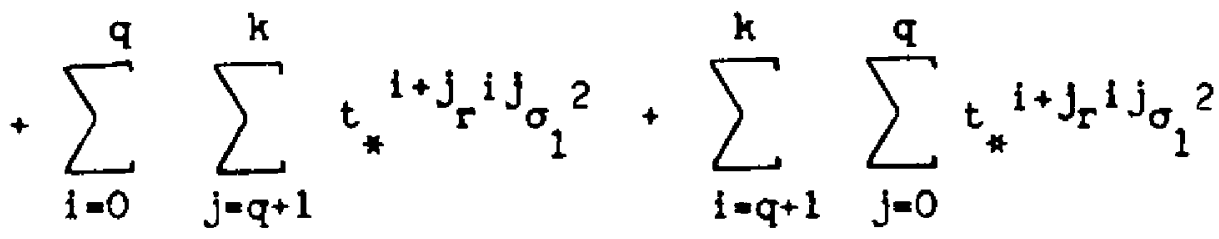




$$
\begin{aligned}
& +\sum_{i=q+1}^{k} \sum_{i=q+1}^{k} t^{i+j} r^{i j_{\sigma_{1}}^{2}} \\
& -\sigma_{1}^{2} \sum_{i=0}^{k} \sum_{j=0}^{k} t_{*}^{i+j}{ }_{F}^{i j}+\sigma_{2}^{2} \sum_{i=0}^{q} \sum_{j=0}^{q} t_{*}^{i+j}{ }^{1 j} \\
& =\sigma_{1}^{2} P_{2 k}\left(t_{*} ; s_{1}, \ldots, s_{N_{1}}\right)+\sigma_{2}^{2} Q_{2 q}\left(t_{*} ; t_{1}, \ldots, t_{N_{2}}\right) \text {, }
\end{aligned}
$$

where $P$ and $Q$ are polynomials in $t_{*}$ of degrees indicated by the subscrlpts and whose coefficients are functions of the observed values of times s's and t's. We now construct a Student-t variate. Observe that $u / \sigma_{u}$ is normally distributed with mean zero and unit variance and that

$$
u=\left(a_{0}+\ldots+a_{k} t^{k}\right)-\left(b_{0}+\ldots+b_{q} t_{*}^{q}\right)
$$

Let

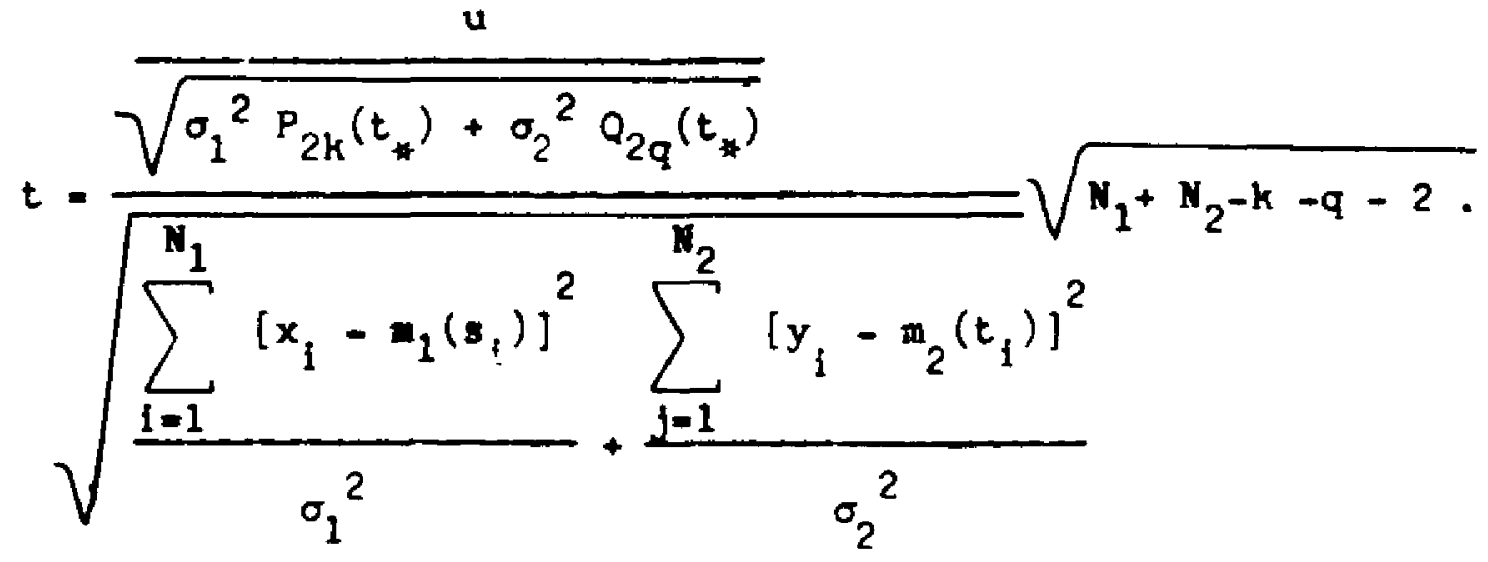

Then $t$ is a Student variate with $\left(N_{1}+n_{2}-k-q-2\right)$ degrees of 
freedom because the numerator is normal $(0,1)$, the denominator is $\sqrt{\chi^{2} / d . f .}$, and the numerator and denominator are inderendent because the terms in the numerator are linear combinations of the a's and b's, which in turn are kncwn to be independent of the sums of squares in the variance estimates.

The $t$ variate constructed has three unknown parameters, $t_{*}$, $\sigma_{1}^{2}$ and $\sigma_{2}{ }^{2}$. This is similar to the situation in the problem of testing the difference between means of two normal populations. The only solution for this more elementary problem is obtained by assuming $\sigma_{1}^{2}=\sigma_{2}^{2}$ or, more generally, $\sigma_{1}{ }^{2}=n \sigma_{2}{ }^{2}$ for some known constant $h$. Making this latter assumption for our problem we have

$$
t=\frac{\frac{u\left(t_{*}\right)}{\sqrt{h^{2} P\left(t_{k}\right)+Q\left(t_{*}\right)}}}{\sqrt{\frac{\sum_{i=1}^{N_{1}}\left[x_{i}-m_{1}\left(s_{i}\right)\right]^{2}}{h^{2}}+\sum_{i=1}^{N_{2}}\left[y_{i}-m_{2}\left(t_{i}\right)\right]^{2}}} \sqrt{1}
$$

This Student variate involves just one parameter $t_{*}$. Confidence linits at significance $c$ are

$$
-t_{\varepsilon / 2}<t<t_{\varepsilon / 2} \text {, or } 0 \leqslant t^{2}<t_{\varepsilon / 2}^{2} \text {. }
$$

Let the denominator radical of $t$ be denoted by $H$. Then by mantpulating the inequality, 


$$
0 \leqq u^{2}\left(t_{*}\right)<\left(h^{2} P\left(t_{*}\right)+Q\left(t_{*}\right)\right) H^{2} t_{t / 2}^{2}
$$

All $t_{*}$ values satisfying the above would constitute the confidence region for all $t_{*}$ values defined by the original polynomial equation. The linits of the regionare obtalned by solving the polynomial equation of degree $2 k$

$$
u^{2}\left(t_{*}\right)=\left[n^{2} P\left(t_{*}\right)+Q\left(t_{*}\right)\right] H^{2} t_{\varepsilon / 2}^{2}
$$

This polynomial will be called the confidence interval polynomial.

If all $2 \mathrm{k}$ roots of the above equation are real, the M.L. estimates of $t_{*}$ are contained in the above confidence set. To see this, note that the region defined by

$$
-t_{\epsilon / 2}<t\left(t_{*}\right)<t_{\epsilon / 2}
$$

has been replaced by an equivalent set of intervals about $t_{*}$. Since $t\left(t_{*}\right)=0$ only if $u\left(t_{*}\right)=0$, then the solutions to $u\left(t_{*}\right)=0$ must 11 ie in the new equivalent sets.

In case $\sigma_{1}{ }^{2}$ and $\sigma_{2}{ }^{2}$ are not known-multiples of each other, it would theoretically be possible to establish a joint three dimensional confidence region for $\left(t_{*}, \sigma_{1}{ }^{2}, \sigma_{2}{ }^{2}\right)$. Consider the event

$$
E:\left[-p<\frac{u\left(t_{*}\right)}{\sigma_{u}\left(t_{*}\right)}<p, q_{1}<\frac{N_{1} \hat{\sigma}_{1}^{2}}{\sigma_{1}{ }^{2}}<q_{2}, q_{3}<\frac{N_{2} \hat{\sigma}_{2}{ }^{2}}{\sigma_{2}{ }^{2}}<q_{4}\right] \text {. }
$$

The three component events are independent; call thes $E_{1}, E_{2}, E_{3}$. Then $P(E)=P\left(E_{1}\right) \cdot P\left(E_{2}\right) \cdot P\left(E_{3}\right)$. If we require $P(E)=.95$ (say), then one way this can be reallzed is to take each $P\left(E_{1}\right)=(.95)^{1 / 3}$. 
Such a specification of the three probabilities defines a three dimensional region in the space of $\left(t_{*}, \sigma_{1}{ }^{2}, \sigma_{2}{ }^{2}\right)$.

The principal result of this section be sumarized in the following theorem.

Theorem. Let $\mu_{1}(t)$ and $\mu_{2}(t)$ be unknown polynomial branch regressions with largest degree $k$, and let $\hat{\mu}_{1}(t)$ and $\hat{\mu}_{2}(t)$ be their maximum likelihood estimators. Let $t_{*}$ be defined by equating $\mu_{1}\left(t_{*}\right)=\mu_{2}\left(t_{*}\right)$ and assume the unknown constant variances of the branches satisfy $\sigma_{1}{ }^{2}=h_{2}{ }^{2}$ for some positive known $h$. Define $u\left(t_{*}\right)=\hat{\mu}_{1}\left(t_{*}\right)-\hat{\mu}_{2}\left(t_{*}\right)$ and consider the equation

$$
u^{2}\left(t_{*}\right)=\left[h^{2} P\left(t_{*}\right)+Q\left(t_{*}\right)\right] \cdot H^{2} t_{1-\varepsilon / 2}^{2},
$$

where $P$ and $Q$ are polynomials in $t_{k}, H^{2}$ is a function of $h$, and $t_{1-c / 2}^{2}$ is a student percentile. A11 of these quantities are previously defined explicitly. If the above equation has $2 k$ real roots, these roots define the end points of $k$ confidence intervals, with joint confidence $1-c$, for the $k$ roots $t_{k}$.

\subsubsection{Expliclt Results for Polynomials of 1st Degree}

Consider the assumptions that the variances are equal, $\sigma_{1}{ }^{2}=\sigma_{2}{ }^{2}=\sigma^{2}$, and that both branch regressions are of first degree. Then $t_{*}$ is defined by the intersection $\mu_{1}=\mu_{2}$,

$$
a_{0}+a_{1} t_{*}=\beta_{0}+\beta_{1} t_{*}
$$

By the theorem in section 2.2 .2 the M.L. estimate $\hat{t}_{*}$ is obtained 
by equating the sample regressions,

$$
\begin{aligned}
a_{0}+a_{1} \hat{t}_{*} & =b_{0}+b_{1} \hat{t}_{*} \\
\hat{t}_{*} & =\frac{b_{0}-a_{0}}{a_{1}-b_{1}}
\end{aligned}
$$

Then according to the previous section, confidence intervals with confidence $\varepsilon$ for $t_{\#}$ are given by the solutions $A$ to the second degree equation

$$
\begin{aligned}
& u^{2}(A)=\left[P_{2}(A)+Q_{2}(A)\right] H^{2} t_{1-\varepsilon / 2}^{2} \\
& {\left[\left(a_{0}-b_{0}\right)+\left(a_{1}-b_{1}\right) A\right]^{2}=t_{1-\varepsilon / 2}^{2} \hat{\sigma}^{2}\left[r^{\infty}+2 r^{01} A+r^{11} A^{2}+\right.} \\
& \left.s^{\infty}+2 s^{01} A+s^{11}{ }_{A}^{2}\right] .
\end{aligned}
$$

But in the solution for the a's and i's (sections 2.1 and 1.6)

$$
\begin{aligned}
& \left(r_{i j}\right)=\left[\begin{array}{cccc}
\Sigma & \Sigma s_{i} \\
\Sigma s_{i} & \Sigma s_{i}{ }^{2}
\end{array}\right] \\
& \left(s_{1 j}\right)=\left[\begin{array}{cc}
\Sigma 1 & \Sigma t_{1} \\
\Sigma t_{1} & \Sigma t_{1}{ }^{2}
\end{array}\right]
\end{aligned}
$$

Hence

$$
\left(r^{1 j}\right)=\frac{1}{\operatorname{det}\left(r_{1 j}\right)}\left[\begin{array}{cc}
\Sigma s_{1}^{2} & -\Sigma s_{1} \\
-\Sigma s_{1} & v_{1}
\end{array}\right]
$$




$$
\begin{aligned}
& \left(s^{1 j}\right)=\frac{1}{\operatorname{det}\left(s_{i j}\right)}\left[\begin{array}{cc}
\Sigma t_{i}^{2} & -\Sigma t_{1} \\
-\Sigma t_{i} & w_{2}
\end{array}\right] \\
& {\left[\begin{array}{l}
a_{0} \\
a_{1}
\end{array}\right]=\left(r^{1 j}\right)\left[\begin{array}{cc}
\Sigma x_{1} \\
\Sigma x_{1} s_{1}
\end{array}\right]} \\
& {\left[\begin{array}{l}
b_{0} \\
b_{1}
\end{array}\right]=\left(s^{1 j}\right)\left[\begin{array}{c}
\Sigma y_{1} \\
\Sigma y_{1} t_{1}
\end{array}\right]} \\
& \hat{\sigma}^{2}=\frac{1}{N_{1}+N_{2}-4}\left[\Sigma\left(x_{1}-a_{0}-a_{1} s_{1}\right)^{2}+\Sigma\left(y_{1}-b_{0}-b_{1} t_{i}\right)^{2}\right] .
\end{aligned}
$$

We now present numerical example.

Example. Consider two normal randon variables $X$ and $Y$ having true branch regressions

$$
\begin{array}{ll}
E[X]=\mu_{1}=2-2 t & t \leq 0 \\
E[Y]=\mu_{2}=2+t & t \geq 0
\end{array}
$$

Obviously $t_{*}=0$. Assume $\sigma_{1}^{2}=\sigma_{2}^{2}$.

Using tables of norml $(0,1)$ random numbers, samples of $X$ and $Y$ were constructed. See Table 1 . The regression analysis was performed

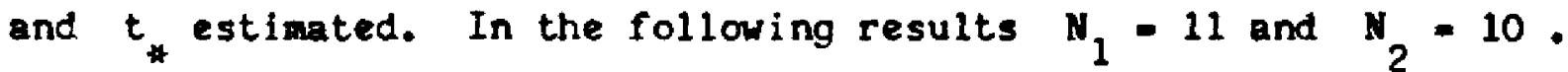

The sample regressions obtained were

$$
\begin{aligned}
& m_{1}-1.01-2.06 t \\
& m_{2}-2.50+0.98 t .
\end{aligned}
$$

Equating $n_{1}=m_{2}$, the point estimate for the abscissa of intersection 
was

$$
\hat{t}_{*}=-0.49
$$

The sample variances vere

$$
\begin{aligned}
& \hat{\sigma}_{1}^{2}=1.1025 \\
& \hat{\sigma}_{2}^{2}=0.5041 \\
& \hat{\sigma}^{2}=0.8212 \quad \text { (pooled estimate from } \sigma_{1}{ }^{2} \text { and } \sigma_{2}{ }^{2} \text { ). }
\end{aligned}
$$

For $c=.05, t_{.975}=2.11$. The equation for confidence 1 imits A was

$$
\begin{aligned}
{[(-1.49)+(-3.04) A]^{2}=} & (2.11)^{2}(.8212)\left(\frac{385}{1210}+\frac{2(55)}{1210} A+\right. \\
& \left.\frac{11}{1210} A^{2}+\frac{385}{825}+\frac{2(-55)}{825} A+\frac{10}{825} A^{2}\right) \\
2.22+9.06 A+9.22 A^{2}= & (4.46)(.8212)\left(.776-.041 A+.130 A^{2}\right) \\
-.62+9.21 A+9.70 A^{2}= & 0
\end{aligned}
$$$$
A=-1.01 \text { and } .07 \text {. }
$$

The numers used in the calculations were obtained from Table 2 .

Now to analyze the results. By taking $N_{1}=11$ and $N_{2}=10$ we are saying that we know $t_{*}$ is in the interval $I:[0,1]$. For this choice of $N_{1}$ and $N_{2}$ the point estimate was $\hat{t}_{\star}=-0.49$, while the $95 \%$ confidence intervals was $\left[-1.01 \leqq t_{*} \leqslant 0.07\right]$. Are these results contradictory or helpful?

The results are extremely helpful because they suggest a way to Incorporate certain types of non-statistical information into an estination procedure. In general (but using current notation) suppose 
we want to estimate a constant $t_{*}$ subject to the known condition that $t_{*}$ is restricted to some interval $I$. Let $\hat{t}_{*}$ denote an

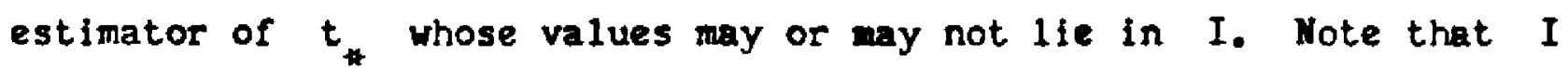
may be regarded as a $100 \%$ confidence interval. Let $J$ denote a randomly determined confidence interval for $t_{\star}$ having confidence $(1-c) 100 \%$. The events "I covers $t_{*}$ " and " $J$ covers $t_{*}$ "are independent. Define $G=I \cap J$. Then

$$
P\left(G \text { covers } t_{*}\right)=(1 .)(1-c)-1-\varepsilon
$$

If the interval $G$ is non-empty it is an improved confidence interval with coefficient $1-\varepsilon$. If $G$ is empty, the situation corresponds to the $\varepsilon$ proportion of the samples for which a confidence interval falls to cover the true paraneter. Another interpretation is that the a priori information that $I$ covers $t_{*}$ is better than the "J covers $t_{*}$ " information obtained from the sample by the M.L. procedures.

A point estimator for $t_{*}$ can now be assoclated with G. From elementary statistics recall that point estimators are usually contained in associated confidence intervals. In fact, if the confidence intervals were somehow derived first, one could define the point estinator as the linit of confidence intervals as their lengths approach zero. This is the logic to be used here, using the improved confidence intervals.

The number $c$ can be taken small enough so that in $J$ is not enpty. Then determine $\tilde{t}_{\#}$ as the liniting abscissa of $I n J$ as (1 - e) appronches zero. Since I is flxed, this value is the 
end point of $J$ closest to the intersection $\hat{t}_{*}$. The estimator for t is thus

$$
E_{*}=\left\{\begin{array}{l}
E_{*} \text { (the M.L. estimate) if } \hat{t}_{*} \text { is in } I \\
\text { end point of } I \text { closest to } \hat{t}_{*} \text { if } \hat{t}_{*} \text { is not in } I \text {. }
\end{array}\right.
$$

It. should be noted that the idea of defining the point estimate as the limit of the imrroved confldence interval produces the M.L. estimate when $\hat{t}_{*}$ is in $I$.

To estirate $x_{*}$, minimize the weighted sum of squared residuals from $\tilde{x}_{*}$ to $\hat{\mu}_{1}\left(\hat{t}_{*}\right)$ and $\hat{\mu}_{2}\left(\hat{t}_{*}\right)$. Thus consider

$$
R=\frac{1}{\sigma_{1}^{2}}\left[\tilde{x}_{*}-\hat{\mu}_{1}\left(\hat{t}_{*}\right)\right]^{2}+\frac{1}{\sigma_{2}{ }^{2}}\left[\ddot{x}_{*}-\hat{\mu}_{2}\left(\hat{t}_{*}\right)\right]^{2}
$$

Differentiating $w$ ith resfect to $x_{*}$, equating to zero and solving gives

$$
\ddot{x}_{*}=\frac{\sigma_{2}^{2}}{\sigma_{1}^{2}+\sigma_{2}^{2}} \hat{\mu}_{1}\left(\tilde{t}_{*}\right)+\frac{\sigma_{1}^{2}}{\sigma_{1}^{2}+\sigma_{2}^{2}} \hat{\mu}_{2}\left(\hat{t}_{*}\right)
$$

If $\hat{t}_{*}$ falls in $I$ this estimate is the abscissa of intersection and hence agrees with M.L. estimator.

Returning to our example we obtain the improved, truncated estimate

$$
\check{t}_{*}=0.0
$$

and the Ieproved $95 \%$ confidence interval

$$
\text { InJ : } 0 \leqslant t \leqslant 0.07
$$

We couldn't expect all results with improved confidence intervals and truncated estimators to be thls good. 
Semple Regression Data

\begin{tabular}{|c|c|c|c|c|c|c|}
\hline \multirow[b]{2}{*}{$\underline{1}$} & \multirow[b]{2}{*}{$\underline{s}_{i} 2 t_{i}$} & \multirow[b]{2}{*}{$\varepsilon_{1} \mathbb{N}(0,1)$} & \multicolumn{2}{|c|}{$\begin{array}{c}\text { Linear Case } \\
k=1\end{array}$} & \multicolumn{2}{|c|}{$\begin{array}{c}\text { Quedratic Case } \\
k=2\end{array}$} \\
\hline & & & $\underline{\underline{ }}$ & $X$ and $Y$ & $\underline{E}$ & $\underline{X}$ and $\mathbf{Y}$ \\
\hline 1 & -10 & -2.052 & 22 & 19.948 & 122 & 119.948 \\
\hline 2 & -9 & -1.378 & 20 & 18.622 & 101 & 99.622 \\
\hline 3 & -8 & 1.453 & 18 & 19.453 & 82 & 83.453 \\
\hline 4 & -7 & 0.355 & 16 & 16.355 & 65 & 65.355 \\
\hline 5 & -6 & -0.636 & 14 & 13.364 & 50 & 49.364 \\
\hline 6 & -5 & -0.577 & 12 & 11.423 & 37 & 36.423 \\
\hline 7 & -4 & -1.026 & 10 & 8.974 & 26 & 24.974 \\
\hline 8 & -3 & 0.124 & 8 & 8.124 & 27 & 17.124 \\
\hline 9 & -2 & -1.299 & 6 & 4.701 & 10 & 6.701 \\
\hline 10 & -1 & -1.094 & 4 & 2.906 & 5 & 3.906 \\
\hline 11 & 0 & -1.697 & 2 & 0.303 & 2 & 0.303 \\
\hline 12 & 1 & 0.798 & 3 & 3.798 & 5 & 5.798 \\
\hline 13 & 2 & -0.331 & 4 & 3.669 & 12 & 11.669 \\
\hline 14 & 3 & 0.446 & 5 & 5.446 & 23 & 23.446 \\
\hline 15 & 4 & 0.759 & 6 & 6.759 & 38 & 38.759 \\
\hline 16 & 5 & 1.673 & 7 & 8.673 & 57 & 58.673 \\
\hline 17 & 6 & -0.341 & 8 & 7.659 & 80 & 79.659 \\
\hline 18 & 7 & -0.534 & 9 & 8.466 & 107 & 106.466 \\
\hline 19 & 8 & 0.637 & 10 & 10.637 & 138 & 138.637 \\
\hline 20 & 9 & 0.054 & 11 & 11.054 & 173 & 173.054 \\
\hline 21 & 10 & 0.661 & 12 & 12.661 & 212 & 212.661 \\
\hline
\end{tabular}


Calculations

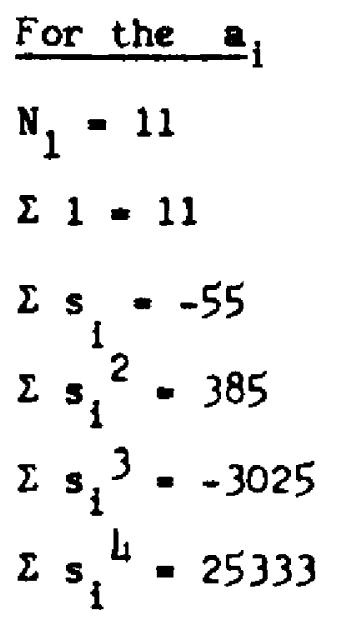

\section{Linear Case}

$$
\begin{aligned}
& \text { For the } b_{i} \\
& \mathrm{~N}_{2}=10 \\
& \Sigma 1=10 \\
& \Sigma t_{i}=55 \\
& \Sigma t_{1}^{2}=385 \\
& \Sigma t_{1}^{3}=3025 \\
& \Sigma t_{i}^{4}=25333
\end{aligned}
$$

$\Sigma x_{1}=124.173$

$z s_{1} x_{1}=-847.062$

$\Sigma s_{1}^{2} x_{1}=6554.658$

$\Sigma x_{i}^{2}=1876.856345$

Quadratic Case
$\Sigma y_{i}=78.822$
$\Sigma t_{i} y_{1}=514.283$
$\Sigma t_{1}^{2} y_{1}=3925.257$
$\Sigma y_{1}^{2}=704.421334$

$\Sigma x_{i}=509.173$

$\sum s_{1} x_{1}=-3872.062$

$\Sigma s_{1}^{2} x_{1}=31877.658$

$\Sigma x_{i}{ }^{2}=40319.172345$
$\Sigma y_{1}=78.822$

$\Sigma t_{i} y_{i}=6564.283$

$\Sigma t_{i}^{2} y_{i}=54591.257$

$\Sigma y_{1}{ }^{2}=117,737.449334$ 


\subsubsection{Explicit Results for Polynomials of 2nd Degree}

Consider the assumptions that the variances are equal, $\sigma_{1}{ }^{2}=\sigma_{2}{ }^{2}=\sigma^{2}$, and that both branch regressions are of second degree. Then $t_{*}$ is defined as the intersection $\mu_{1}=\mu_{2}$ :

$$
a_{0}+a_{1} t_{*}+a_{2} t^{2}-\beta_{0}+\beta_{1} t_{*}+\beta_{2} t^{2}
$$

The point estimate is obtained by equating the sample regressions

$$
a_{0}+a_{1} \hat{t}_{*}+a_{2} \hat{t}_{*}^{2}=b_{0}+b_{1} \hat{t}_{*}^{2}
$$

Confidence linits with confidence $1-\varepsilon$ for both roots are obtained from the solutions $A$ of the fourth degree polynomial equation (with $\left.c_{j}=a_{j}-b_{j}\right)$

$$
\begin{aligned}
{\left[c_{0}+c_{1} A+c_{2} A^{2}\right]^{2}=} & t_{1-\varepsilon / 2}^{2} \hat{\sigma}^{2} \\
& {\left[\left(r^{00}+s^{00}\right)+\left(r^{11}+s^{11}\right) A^{2}+\right.} \\
& \left(r^{22}+s^{22}\right) A^{4}+2\left(r^{02}+s^{01}\right) A+ \\
& \left.2\left(r^{02}+s^{02}\right) A^{2}+2\left(r^{12}+s^{12}\right) A^{3}\right] .
\end{aligned}
$$

In this case, with summations fros 1 to $N_{1}$

$$
\left(r_{i j}\right)=\left[\begin{array}{lllll}
\Sigma 1 & \Sigma s_{i} & \Sigma s_{i}^{2} \\
\Sigma s_{i} & \Sigma s_{i}^{2} & \Sigma s_{i}{ }^{3} \\
\Sigma s_{i}^{2} & \Sigma s_{i}^{3} & \Sigma s_{i}
\end{array}\right]
$$

Simllarly the matrlx $\left(s_{i j}\right)$ is formed from the observed $t$ 's, where the sumations would run from 1 to $\mathbb{N}_{2}$. 
From the theory of determinants,

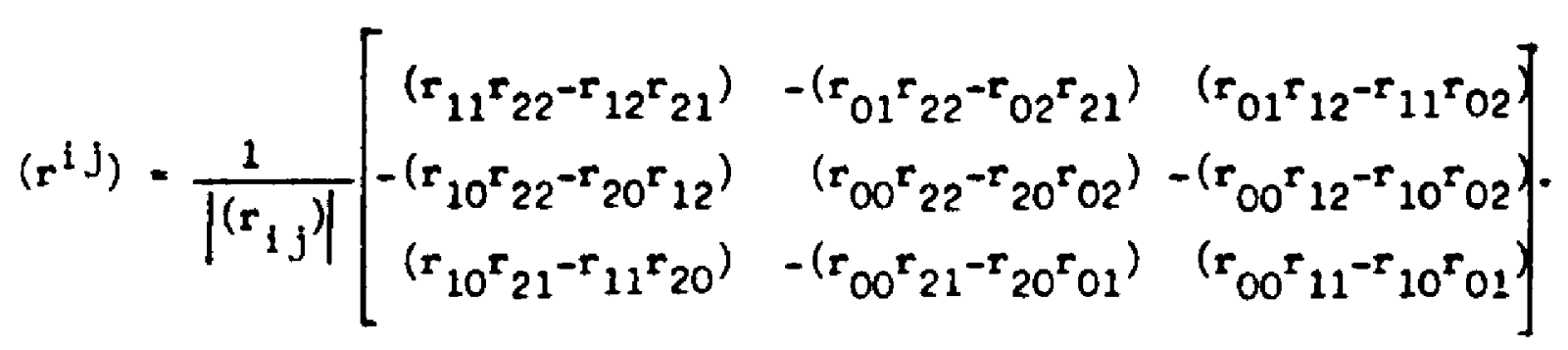

Similarly $\left(s^{i j}\right)$ is obtained using the elements of $\left(s_{i j}\right)$.

Then

$$
\left[\begin{array}{l}
a_{0} \\
a_{1} \\
a_{2}
\end{array}\right]=\left[r^{i j}\right]\left[\begin{array}{c}
\Sigma x_{1} \\
\Sigma x_{1} s_{i} \\
\Sigma x_{1} s_{i}^{2}
\end{array}\right] \text { and }\left[\begin{array}{l}
b_{0} \\
b_{1} \\
b_{2}
\end{array}\right]=\left[s^{i j}\right]\left[\begin{array}{c}
\Sigma y_{i} \\
\Sigma y_{1} t_{1} \\
\Sigma y_{i} t_{1}^{2}
\end{array}\right] \text {. }
$$

The unbiased variance estimate is given by

$$
\hat{\sigma}^{2}-\frac{1}{N_{1}+N_{2}-6}\left\{\sum_{i=1}^{N_{1}}\left[x_{1}-\mu_{1}\left(s_{i}\right)\right]^{2}+\sum_{i=1}^{n_{2}}\left[y_{i}-\mu_{2}\left(t_{1}\right)\right]^{2}\right\} .
$$

Example. Let the true regressions for $X_{3}$ and $Y_{t}$ be

$$
\begin{aligned}
& \mu_{1}=2-2 t+t^{2} \quad t \leq 0 \\
& \mu_{2}=2+t+2 t^{2} \quad t>0 \text {. }
\end{aligned}
$$

Obviously $t_{*}=0$. The true variance is one, but this is assumed unknown.

Consider the sample of size $\mathbf{M}=21$ given in Table 1 . Then with $N_{1}=11$ ( the $s_{1}$ are $\left.-10,-9, \ldots,-1,0\right)$ and $N_{2}=10$ :

$$
\left(r_{1 j}\right)=\left[\begin{array}{rrr}
11 & -55 & 385 \\
-55 & 385 & -3025 \\
385 & -3025 & 25333
\end{array}\right] \text {. }
$$


Using the results in Table 2 we obtain

$$
\begin{aligned}
& \operatorname{det}\left(r_{i j}\right)=1038180 \\
& \left(r^{1 j}\right)=\frac{1}{1038180}\left[\begin{array}{rrr}
602580 & 228690 & 18150 \\
228690 & 130438 & 12100 \\
18150 & 12100 & 1210
\end{array}\right] \text {. }
\end{aligned}
$$

In a similar manner

$$
\left(s_{i j}\right)=\left[\begin{array}{rrr}
10 & 55 & 385 \\
55 & 385 & 3025 \\
385 & 3025 & 25333
\end{array}\right]
$$

$$
\begin{aligned}
\operatorname{det}\left(s_{i j}\right) & =435600 \\
\left(s^{i j}\right) & =\frac{1}{435600}\left[\begin{array}{rrr}
602580 & -228690 & 18150 \\
-228690 & 105105 & -9125 \\
18150 & -9125 & 825
\end{array}\right] .
\end{aligned}
$$

From these results we obtain

$$
\begin{aligned}
& \hat{\mu}_{1}(t)=-0.101-2.795 t+0.926 t^{2} \\
& \hat{\mu}_{2}(t)=2.591+0.933 t+2.004 t^{2} \\
& \hat{\sigma}^{2}=0.9631
\end{aligned}
$$

Equating the sample regressions and solving gives

$$
t_{*}{ }^{(1)}=-0.54 \quad \text { and } \quad t^{(2)}=-2.4
$$

Let $c=.05 ;$ then $t_{.975}=2.11$. The equation defining 
end points $A$ for the confidence intervals is

$$
\begin{aligned}
& {\left[2.692+3.728 A+1.078 A^{2}\right]^{2}=(2.11)^{2}(.9631)} \\
& {\left[0.5804+0.4406 A+0.1256 A^{2}+0.0234 A^{3}+0.0012 A^{4}\right.} \\
& \left.+1.3833-1.0500 A+0.2413 A^{2}-0.0416 A^{3}+0.0019 A^{4}\right],
\end{aligned}
$$

or

$$
-1.1730+22.6846 A+17.5781 A^{2}+8.1157 A^{3}+1.1489 A^{4}=0 .
$$

The solutions obtained are

$$
A=0.06, \quad-5.42, \quad \text { and two complex roots. }
$$

As in the linear example the situation arlses where the M.L. estimator doesn't fall in the known interval $I$, which in this case is $[0,1]$. Nevertheless these resulis provide a great deal of valuable inforwation. The graph of $u(A)=0$ is of the nature given in figure 2 . The graph of $u^{2}(A)=0$ is therefore of the nature given in figure 3 . By equating $u^{2}(A)$ to a positive polynomial, as our derivation requires, we obtaln

$$
u^{2}(A)=\left[h^{2} p+Q\right] \cdot H^{2} t^{2}
$$

This function is show in figure 3 . For the above example it is geonetrically clear that this equation defines two complex roots and hence the real roots obtained must be a lower bound for the smallest root and an upper bound for the largest root. Thus the $95 \%$ confidence intervals are undefined as closed intervals. Instead we obtain two $97.5 \%$ one-sided confidence intervals: $(-5.4, \infty)$ for $t_{*}(2)$ and $(-\infty, 0.06)$ for $t_{*}^{(1)}$. Using the concepts introduced 
37

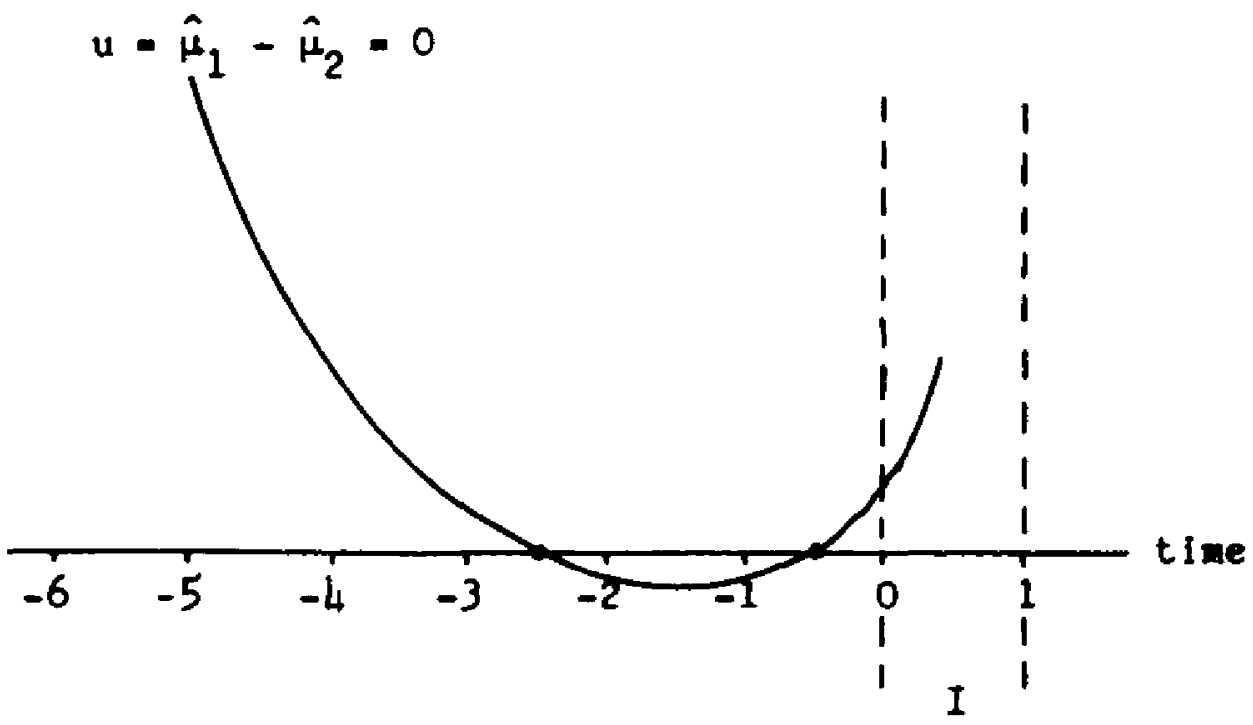

Flgure 2

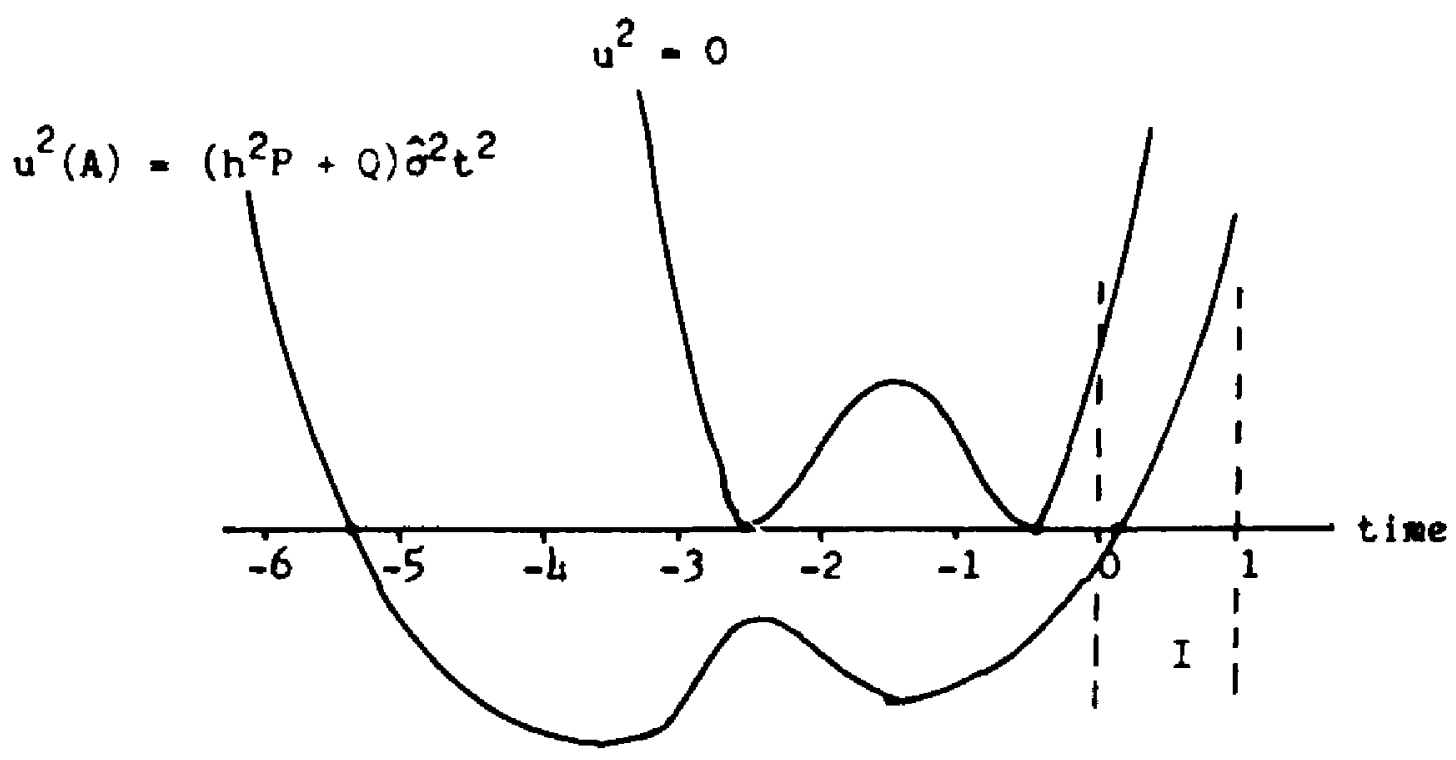

Figure 3 
In section 2.3 .1 we readily obtain the $97.5 \%$ improved confidence interval $(0,0,0.06)$. The associated point estimate is $E_{*}=0.0$.

\subsubsection{General Theorem for Polynonials of k-th Degree}

The results derived from the previous two examnles can be sumarized and extended to any degree polynomial assumptions in the following procedural theoren.

Theorem. Let $\hat{t}_{*}$ be a solution to the equation $\hat{\mu}_{1}\left(\hat{t}_{*}\right)-\hat{\mu}_{2}\left(\hat{t}_{*}\right)$ and suppose $\hat{t}_{*}$ can be identified with a true value $t_{*}$ known to be in $I$, the interval between the sample of $\left(x_{1}, \ldots, x_{\mathrm{N}_{1}}\right)$ and the sample of $\left(y_{1}, \ldots, y_{N_{2}}\right)$. For $c$ sufficiently small construct a confidence interval $\mathrm{J}$ which intersects $I$. Then $I n J$ is a (1-E) $100 \%$ confidence interval for $t_{*}$, and the limit of InJ as $\varepsilon$ approaches 1 defines a point estimator $E_{*}$. Let

$$
\tilde{\mathbf{x}}_{*}=\frac{\hat{\sigma}_{2}^{2}}{\hat{\sigma}_{1}^{2}+\hat{\sigma}_{2}^{2}} \hat{\mu}_{1}\left(\tilde{t}_{*}\right)+\frac{\hat{\sigma}_{1}^{2}}{\hat{\sigma}_{1}^{2}+\hat{\sigma}_{2}^{2}} \hat{\mu}_{2}\left(\hat{t}_{*}\right) \text {. }
$$

If $\hat{t}_{*}$ falls in I then $\left(\hat{t}_{*}, \tilde{x}_{*}\right)$ are the maximum likelihood estimators for $\left(t_{*}, x_{*}\right)$. If $\hat{t}_{*}$ does not fall in $I$ then $\left(\tilde{t}_{*}, \tilde{x}_{*}\right)$ are merely truncated estinates of $\left(t_{*}, x_{*}\right)$.

This solution require a detalled study of the $t_{*}$-estimator polynomial in conjunction with the confidence interval polynomial in order to deteralne which boundaries correspond to which solutions. If difficulties are still encountered, consult section 2.4 .3 . 
2.I Discussion of Practical Difficulties

The two theorems of sectlons 2.2 and 2.3 will suffice in many of the practical problems which arise. Yet, the difficulties encountered in the most recent example are symptomatic of difficulties which could occur in (but hopefully) anority of the problems. We shall discuss these problems together with potential remedies.

It is not possible to foresee all difficulties which might arise, but at least partial list can be given:

1) $\hat{\mu}_{1}$ and $\hat{\mu}_{2}$ have more than one intersection in $I$

2) $\hat{\mu}_{1}$ and $\hat{\mu}_{2}$ have real intersections, but none in $I$

3) $\hat{\mu}_{1}$ and $\hat{\mu}_{2}$ have no real intersections at all

4) the confidence interval polynomial fails to have $2 k$ real roots, and/or the real roots which do occur are not readily identifiable with any intersection point $t_{*}$.

\subsubsection{Examination of the Assumptions and the Sample}

If difficulties do arise, some general inquiry should first be made.

Is our likelihood formulation correct? In view of ear1ler discussions, the formulation is correct.

Are our assumptions correct? Are our true regressions polynomials? If not, this may be our source of difficulty and we should then study regression formulatlons not involving polynomials. Since the M.L. estinator requires extrapolation into an interval $I$, it could be that even though a polynomial approximation is sufficiently 
accurate in the given interval of $s^{\prime} s$ and $t$ 's, that it is inaccurate in I. To decrease such chances of fallure, only low degree polynomials should be used. It could be that polynomial assumptions are adequate but that we have hypothesized the wrong degree polynomial. The degree can readily be changed. Note that odd degree polynomials will at least guarantee one real intersection.

If we are satisfled that our assumptions are valid enough, then the other source of difficulty must be the sample. If the sample regressions just barely fall to intersect, it could be that one entire set of data, either the $x^{\prime} s$ or $y^{\prime} s$ ( or, in fact, both), is in error by a constant bias arising from the method of measurement. This situation should be studied and, if true, remelied. Otherwise the entire sample may be bad. Recalling that every sample value has the typical form

$$
x_{1}=\mu_{1}\left(s_{1}\right)+\varepsilon_{1}
$$

this means that the collection of $\varepsilon^{\prime} s$ is unrepresentative of a normal population with zero mean. An attempt could be made to average out this difficulty. Using $\hat{\sigma}_{1}{ }^{2}$ as a guide, construct a normal sample $\left(\lambda_{1}\right)$ of size $n_{1}$ having zero means and (say) variance equal to $0.5 \hat{\sigma}_{1}^{2}$. Then calculate an adjusted sample

$$
x_{i}{ }^{\prime}=x_{i}+\lambda_{1}, i-1, \ldots, N_{1}
$$

The new sample admittedly has larger variance but it may also produce a $\hat{\mu}_{1}^{\prime}$ which would intersect with $\hat{\mu}_{2}$ in or near enough to $I$. This technique could not be used repeatedly because the variance 
estimates would grow too large; but one or two trials might produce desirable results. An attempt could also be made to edit the sample and remove observations producing significantly large residuals from $\hat{\mu}_{1}$ or $\hat{\mu}_{2}$. Then new estimates of $\mu_{1}$ and $\mu_{2}$ should be calculated from the reduced sample and the M.L. procedure performed again. In edfting one must decide with some certainty that the observations being removed are indeed unrepresentative of the sample and not suggestive of some unforeseen change of events in the entire process.

The fact that difficulties arlse is not altogether surprising when we consider that the estimator for $t_{*}$ is based upon extrapolation. From a prediction standpoint we are thus working outside the most favorable reglons, namely those intervals of definition for the members in the sample. Furthermore, in each of the two examples cited, the true solution was an end-point of $I$. We would expect estimates to fall on either side of $t_{*}$. so when our estimator falls berely on the left of $t=0$ (In the examples) and hence outside $I$, it seems bad but actual ly is not surprising. The cause of our difficulty is thus the bed sample. In simpler estimation problems a maximum likelihood frocedure may well give a bad value of a good estimator. In our estimation problem the H.L. procedure may fail to given any value (In case of sufficiently bad samples) for an otherwise good estimator.

If after study of the formulation, assumption and the sample it is felt that nothing more can be done with these aspects of the situation, and that we must "live" with our sample results, good or bad, the problem is now how to derive the maximum amount of 
information in view of the potential difficulties 1) to 4).

2.4.2 More Than One Solution in I

Suppose that wore than one real solution, $\hat{t}_{*}$, is found in $I$. Since $L$ has already been aximized (and Independently of I) there is no point in checking each $\hat{t}_{*}$ to see which glves the largest $L$. There is nothing to check. In the absence of additional Information concerning which root to choose, some elementary random selection scheme should be used to select a $\hat{t}_{*}$.

\subsubsection{Real Solutions, But None in I}

This probled has been partially discussed in section 2.3 for the case when an estimate $\hat{t}_{*}$ falling outside $I$ is sure to correspond to a true $t_{*}$ in $I$ and when the appropriate confidence intervals can be constructed. However, it could happen that the $\hat{t}_{*}$ obtained is clearly not assoclated with any $t_{*}$ in $I$ (as in figure 4 with degree 3 for the estimator polynomial) or that the confidence intervals could not be constructed (as in figure 5 with degree 6 for the confldence interval polynomlals.

Thus, for our interests there are effectively no real roots at a11. Alternative procedures for this section will therefore be treated in the next section.

\subsubsection{No Real Solutions in I}

Suppose that either there are no real solutions $\hat{t}_{*}$ at $a 11$, or that whatever real solutions are found clearly do not correspond to 


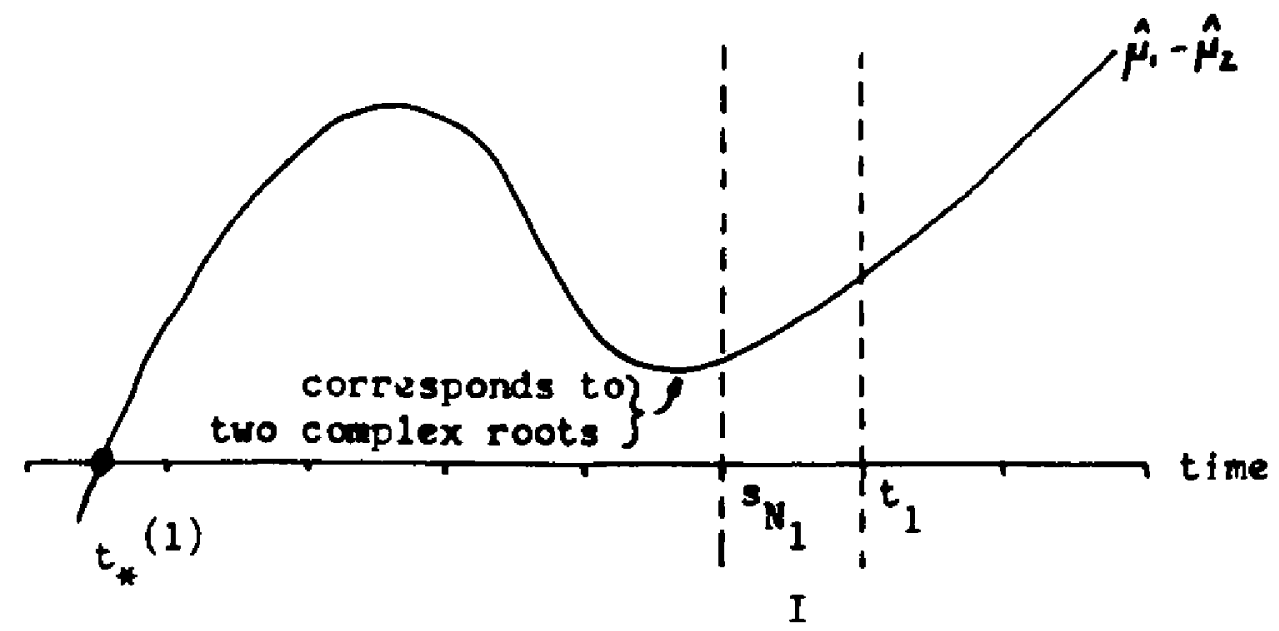

Figure 4

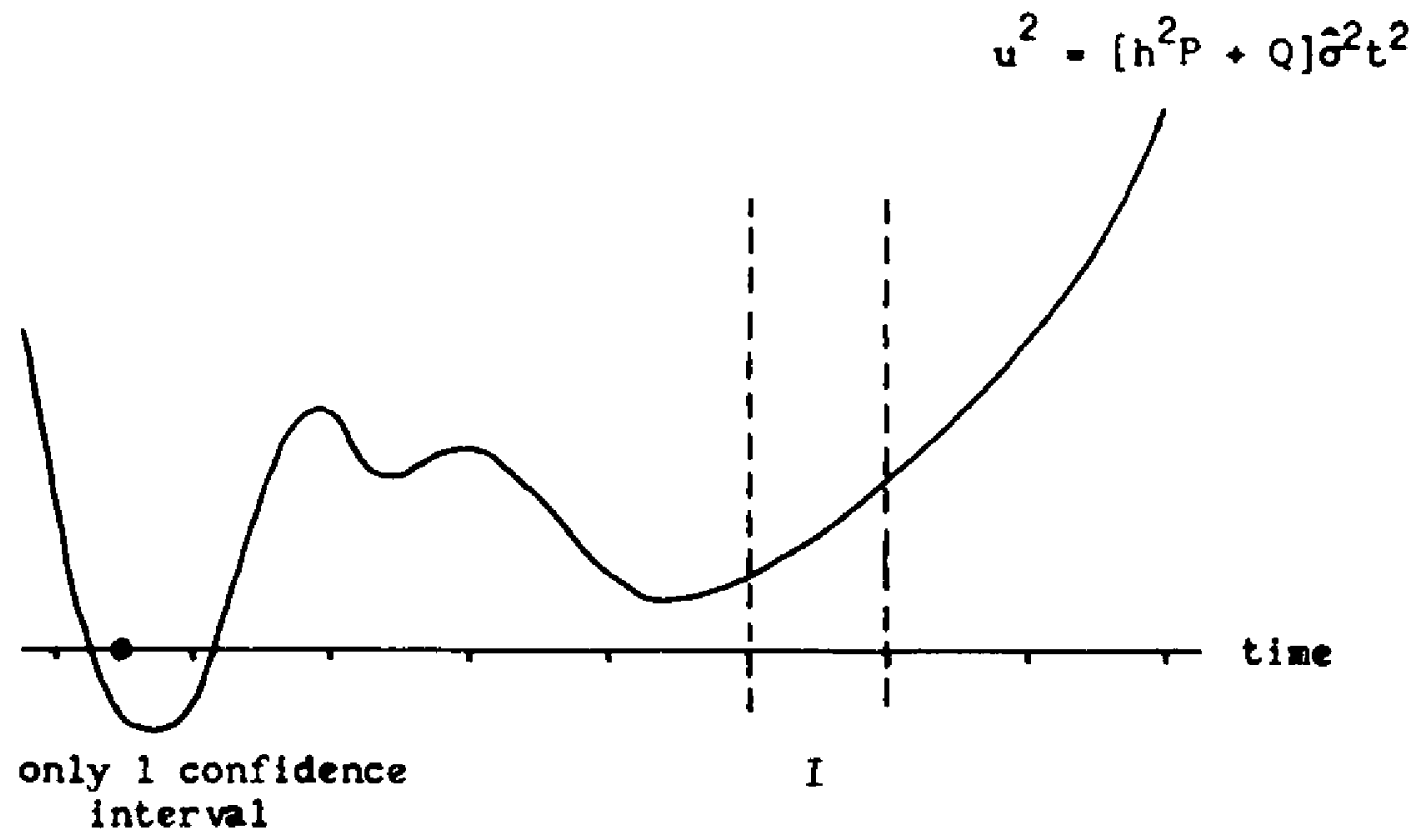

Figure 5 
a true $t_{*}$ in $I$. Our approach will be to define an estimation procedure which will agree with maximum likelihood procedure when M.L. techniques produce a useful $\hat{t}_{*}$ and which 111 otherwise produce a sensible estimate wh desirable properties. The intention is to procede in the spirit of M.L., though not quite in fact. Consider the region $R$ contained between the vertical ines $t=s_{y_{1}}$ and $t=t_{1}$ as Illustrated in figure 6 . Consider the plausibility of seeking as a solution a point $P\left(\hat{t}_{*}, \tilde{x}_{*}\right)$ which maximizes an associated 1 kelihood type function

$$
L_{*}=L \cdot \frac{1}{\sqrt{2 \pi} \sigma_{1}} e^{-\frac{1}{2 \sigma_{1}{ }^{2}}\left[x_{*}-2 a_{j} t_{*}\right]^{2}} \frac{1}{\sqrt{2 \pi} \sigma_{2}} e^{-\frac{1}{2 \sigma_{2}{ }^{2}}\left[x_{*}-\Sigma \beta_{j} t_{*}\right]^{2}}
$$

The definition of $L_{*}$ is motivated by the thought of finding an optinal point $P$ which could have been included in the estimnte of both $\mu_{1}$ and $\mu_{2}$. This idea will be referred to as the guasilikelihood criterion, (Q.L.). The Q.L. function $L_{*}$ is the ordinary M.L. function $L$ multiplied by two distribution factors of the unknown point $\left(t_{*}, x_{*}\right)$; Q.L. estimates w111 typically be denoted $\grave{a}_{1}$. The sole purpose of $Q . L$. is to obtain a method of estimating $\left(t_{*}, x_{*}\right)$ and not to get better estimates of $t$ he $a^{\prime} s$, $\beta^{\prime} s$ and o's. The M.L. estimates for the latter parameters always exist and cannot be improved upon. For Interpolation prediction the M.L. estimates should always be used, not the Q.L. estimates.

Treating '. as a 11 kelihood function, the estimates for $\left(a_{0}, \ldots, \beta_{k}, \sigma_{1}^{2}, \sigma_{2}^{2}, t_{*}, x_{*}\right)$ can be obtained by $\operatorname{maximizing} \log L_{*}$. 


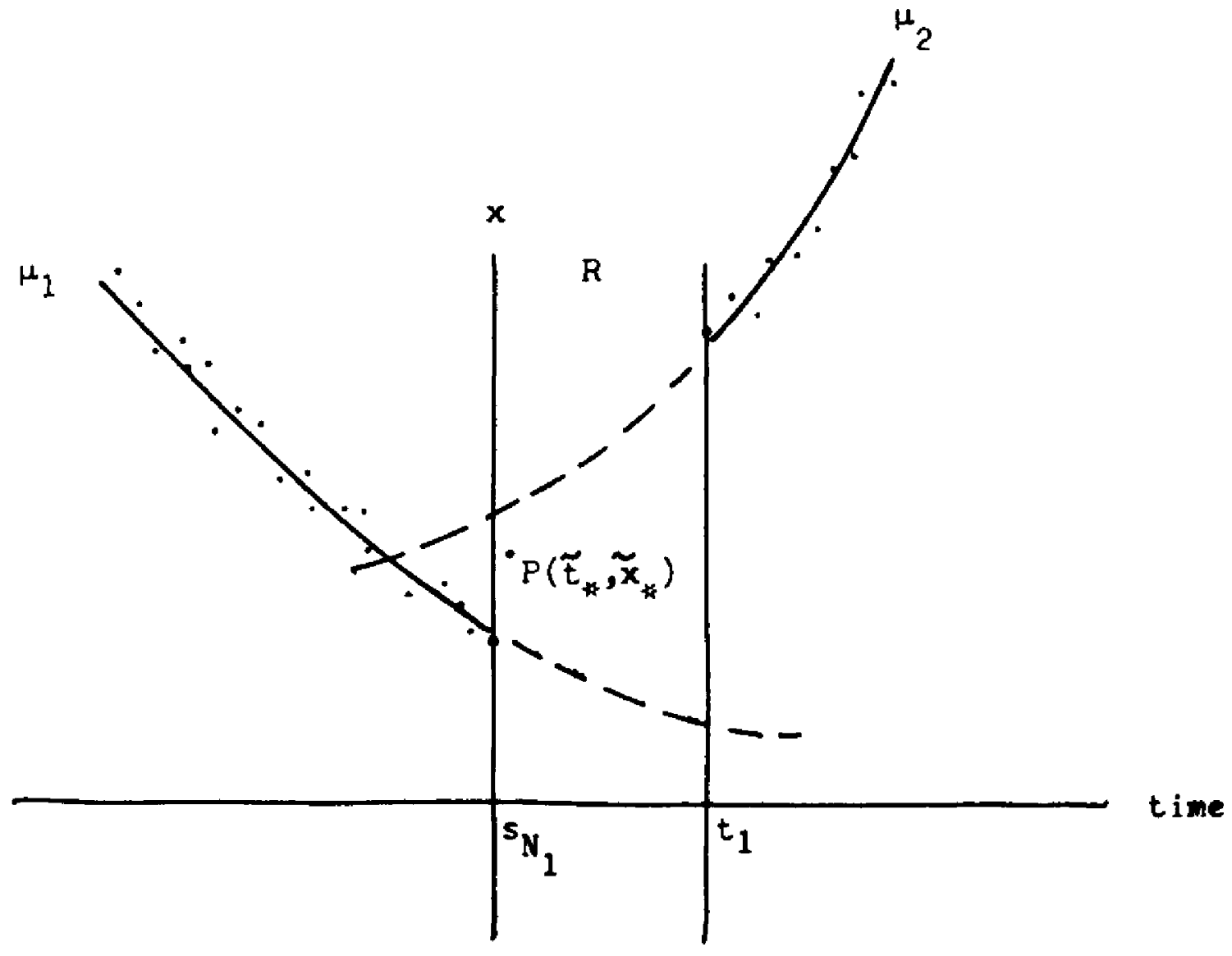

Figure 6 
But looking ahead, the estiates of $\sigma_{1}{ }^{2}$ and $\sigma_{2}{ }^{2}$ would involve the unknown $\left(E_{*}, \tilde{x}_{*}\right)$. This leads to serious computational difficulties because the $\hat{x}_{\#}$ in turn depends on the $\sigma^{\prime} s$. A reasonable alternative is to use the M.L. estimates (which don't involve $t_{*}$ or $\tilde{x}_{*}$ ) for $\sigma_{1}$ and $\sigma_{2}$. Thus the $\sigma^{\prime} s$ in $L_{*}$ are regarded as known constants (as the M.L. estimates). Then finding $\log L_{*}$ and differentlating gives the following system, where $r=0,1, \ldots, k$ for the $a^{\prime} s$ and $r=0,1, \ldots, q$ for the $\beta^{\prime} s$.

$$
\begin{aligned}
\log L_{*}= & -N_{1} \log 2 \pi-N_{1} \log \sigma_{1}-\frac{1}{2 \sigma_{1}^{2}} \sum_{i=1}^{N}\left[x_{i}-\sum_{j} a_{j} s_{i}^{j}\right]^{2} \\
& -N_{2} \log 2 \pi-N_{2} \log \sigma_{2}-\frac{1}{2 \sigma_{2}^{2}} \sum_{1=1}\left[y_{1}-\sum_{j} \beta_{j} t_{1}^{j}\right]^{2} \\
& -\log 2 \pi-\log \sigma_{1}-\frac{1}{2 \sigma_{1}^{2}}\left[x_{*}-\sum \sigma_{j} t_{*}\right]^{2} \\
& -\log 2 \pi-\log \sigma_{2}-\frac{1}{2 \sigma_{2}^{2}}\left[x_{*}-\sum \beta_{j} t_{*}^{j}\right]^{2}
\end{aligned}
$$

(1) $\frac{\log L_{*}}{\partial a_{r}}=-\frac{1}{2 \sigma_{1}^{2}} \sum 2\left[x_{1}-\sum \alpha_{j} s_{1}^{j}\right]\left(-s_{1}^{r}\right)-\frac{1}{2 \sigma_{1}^{2}} 2\left[x_{*}-\sum a_{j} t_{*}^{J}\right]\left(-t_{*}^{r}\right)=0$

(2) $\frac{\log L_{*}}{\partial \beta_{r}}=-\frac{1}{2 \sigma_{2}^{2}} \sum^{2}\left[y_{i}-\sum \beta_{j} t_{1}^{j}\right]\left(-t_{1}{ }^{r}\right)-\frac{1}{2 \sigma_{2}^{2}} 2\left[x_{*}-\Sigma \beta_{j} t_{*}^{j}\right]\left(-t_{*}^{r}\right)=0$

(3) $\frac{\log L_{*}}{\partial x_{*}}=-\frac{1}{2 \sigma_{1}^{2}}(2)\left[x_{*}-\Sigma \alpha_{j} t_{*}^{j}\right]-\frac{1}{2 \sigma_{2}^{2}}(2)\left[x_{*}-\Sigma \beta_{j} t_{*}^{j}\right]=0$

(4) $\frac{\log L_{*}}{\partial t_{*}}=-\frac{1}{2 \sigma_{1}{ }^{2}} \cdot 2\left[x_{*}-\Sigma \alpha_{j} t_{*}^{j}\right]\left(-\Sigma j \alpha_{j} t_{*}^{j-1}\right)-\frac{1}{2 \sigma_{2}{ }^{2}} 2\left[x_{*}-\Sigma \beta_{j} t_{*}^{j}\right] \Sigma-j \beta_{j} t_{*}^{j-1}$ 
Frow (3) the estimate for $\tilde{x}_{*}$ is immediate.

$$
\begin{aligned}
{\left[\frac{1}{\sigma_{1}^{2}}+\frac{1}{\sigma_{2}^{2}}\right] \hat{x}_{*} } & =\frac{1}{\sigma_{1}^{2}} \tilde{\mu}_{1}\left(\tilde{t}_{*}\right)+\frac{1}{\sigma_{2}^{2}} \tilde{\mu}_{2}\left(\tilde{t}_{*}\right) \\
\hat{x}_{*} & =\frac{\hat{\sigma}_{2}^{2}}{\sigma_{1}^{2}+\sigma_{2}^{2}} \tilde{\mu}_{1}\left(\tilde{t}_{*}\right)+\frac{\sigma_{1}^{2}}{\sigma_{1}^{2}+\sigma_{2}^{2}} \tilde{\mu}_{2}\left(\tilde{t}_{*}\right) .
\end{aligned}
$$

This solution for $\tilde{x}_{*}$ requires that $\tilde{t}_{*}$ and ailthe $\alpha$ 's and $\beta$ 's be known. If $\sigma_{1}=\sigma_{2}$ then $\tilde{x}_{*}$ is the numerlcal average of the two regressions $\hat{\mu}_{1}$ and $\hat{\mu}_{2}$ at any absclssa. If the sample regressions intersect then $\tilde{x}_{*}$ is the ordinate of this intersection. Note that (3) is of the form $A+B=0$. One possible solution for this is $A=B=0$. This means that (1) and (2) reduce to the original M.L. normal equations. This is not surprising since $\left(\tilde{t}_{*}, \tilde{x}_{*}\right)$ contribute exactly zero to the various sums of squares. Thus the Q.L. estimates are the M.L. estimates and $E_{\#}$ is obtained as the intersection of the sample regressions if the intersection is in $R$. On the other hand if the sample regression intersects in $R$, then necessarily $A=B=0$. This result, which serves as a consistency check on the value of Q.L. formulation, is sumarlzed in the following theorem.

Theorea. The quasi-likelihood estimate for $\left(t_{*}, x_{*}\right)$ is identical to the maximum likelihood estimate for $\left(t_{*}, x_{*}\right)$ if and only if the sample regressions intersect in $R$, a vertical region of known confinement for $t_{\star}$.

The other possible solution for (3) is that $A+B=0$ with 
neither being zero. This corresponds to non-intersecting sample regressions. In this case, and in the case where $E_{*}$ obviously does not correspond to $R$, we are virtually forced to maximize $L_{*}$ by trial and error. Th!s means that a cholce of $\left(\tilde{t}_{*}, \tilde{x}_{*}\right)$ be mode, $a^{\prime}, 3$ and $\beta^{\prime}$ 's calculated, and the corresponding value of $L_{*}$ be recorded. It is equivalent and easier to record merely

$$
H=\sigma_{2}{ }^{2}\left[\tilde{x}_{*}-\hat{\mu}_{1}\left(\tilde{t}_{*}\right)\right]^{2}+\sigma_{1}^{2}\left[\tilde{x}_{*}-\tilde{\mu}_{2}\left(\bar{t}_{*}\right)\right]^{2} .
$$

These operations would have to be performed over an entire grid work of reasorable points in $R$. A first reasonable choice might well be the abscissa in $R$ where the M.L. regressions $\hat{\mu}_{1}$ and $\hat{\mu}_{2}$ are closest, with $\bar{x}_{*}$ calculated from (3). If near stabilization of the graphs of the H's is observed, then the $t_{*}$ in $R$ corresponding to swooth or boundary minima of $H$ should be tried. After all, $L_{\text {* }}$ is a polynomial in $\hat{t}_{*}$, and the actual $\tilde{t}_{*}$ must occur at smooth extrema or boundary abscissa of $R$. In fact the Q.L. estimation problem for $t_{*}$ is that of finding a point which whe $n$ used in the estimation of Q.L. regressions will produce an $H$ function having that very point as an extrem. It seens geometrically obvious that at least one such point should exist in $R$, but finding it leads to the difficulties ve have just discussed. Eventially the point producing the suallest $H$ is to be taken as (an approximation to) the Q.L. estimate.

It wight well be asked: 'why bother with the Q.L. estimates... why not merely use an abscissa in $R$, call it $t_{c}$, where $\hat{\mu}_{1}$ and $\hat{\mu}_{2}$ are closest?' The answer involves refinement, since a little 
reflection reveals a shortcoming of the latter suggestion. Suppose that all observations $\left(x_{1}, \ldots, x_{N_{1}}, y_{1}, \ldots, y_{N_{2}-1}\right)$ except $y_{N_{2}}$ are error free and lie on $\mu_{1}$ and $\hat{\mu}_{2}$ respectively. Then $\hat{\mu}_{2}$ can be changed merely by raising and lowering $\mathrm{y}_{\mathrm{N}_{2}}$ and can thus be made to intersect $\mu_{1}$ in $R$ at an abscissa denoted by $\hat{\hat{t}}_{\text {* But in }}$ manipulating $y_{N_{2}}$ all sample regressions coefficients ( $b_{0}, \ldots, b_{q}$ )

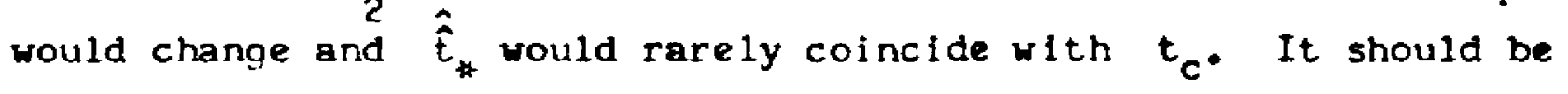
noted that if the sample regressions intersect, then this method of closest regression recovers the $M_{0} L$. estimates.

In summary, the justification for using the Q.L. procedure are therefore (1) that Q.L. reduces to M.L. when $P$ is in $R$, (2) that Q.L. is formulated by a logic similar to M.L., and (3) that Q.L. seems better than the computationally easier method of closest regressions in $R$.

No final distribution theory is yet available for the Q.L. estimator other than when Q.L. and M.L. agree.

\subsubsection{The Method of Intersecting Confidence Regions}

Another idea investigated was that of constructing confidence bands $B_{1}$ and $B_{2}$ of equal confidence around the M.L. regressions $\hat{\mu}_{1}$ and $\hat{\mu}_{2}$ and then taking the intersection $C$ of $B_{1}, B_{2}$, and $R$ ( a known region of confinement for the true $P\left(t_{*}, x_{*}\right)$ ) as a confidence region for $\left(t_{*}, x_{*}\right)$. A point estimate could then be defined as the limit of $C$ as the comon confidence of $B_{1}$ and $B_{2}$ goes to zero.

This method was tried on the 1 inear and quadratic examples of 
section 2.3 . The results were not th a 11 unreasonable and are shown in figures 7 and 8 .

This method was not investigated further since it seems that for larger degree $k$ the confidence bands $B_{1}$ and $B_{2}$ (which are generallzations of hyperbolas) might double back too rapidly and hence not always define a bounded intersection $C$. The bands $B_{1}$ and $B_{2}$ tend to shy away from $\hat{\mu}_{1}$ and $\hat{\mu}_{2}$ at the end points, but that is sub-region where we are most interested.

In this method, if $C$ were bounded and if the sample M.L. regressions were to intersect in $R$, then this estimate for $\left(t_{*}, x_{*}\right)$ would agree $w_{i t h}$ the M.L. estimate. 2.5 A Jump at the Intersection

In the previous section we have assumed that the branch regressions intersect so that $t_{*}$ Is defined as the abscissa such that $\mu_{1}\left(t_{*}\right)=\mu_{2}\left(t_{*}\right)$. In cases where $\mu_{1}$ and $\mu_{2}$ differ by a constant $\lambda$ at $t_{*}$ there may or may not be a solution.

If $\lambda$ is unknown, the problem is anblguous because $t_{\text {* cannot be }}$ uniquely defined. Any $t$ value in the interval $I:\left[s_{N_{1}}, t_{N_{1}+1}\right]$ will have a $\lambda$ value corresponding to it and thus any $\left(t, \lambda_{t}\right)$ would define a $t_{\star}$ * If $\lambda$ is known, there may or may not be a solution, depending on whether anique $t_{\text {* }}$ can be defined. It is geometrically obvious that if $\mu_{1}$ and $H_{2}$ are extrapolated throughout the subdomain $R$, and if in I the two branches are non-intersecting but parallel over any portion of $1 t$, then no unique $t_{*}$ can be defined. If the two branches behave differentiy in $I$, then $t_{*}$ is defined by $\mu_{1}\left(t_{*}\right)=\mu_{2}\left(t_{*}\right)+\lambda$. 


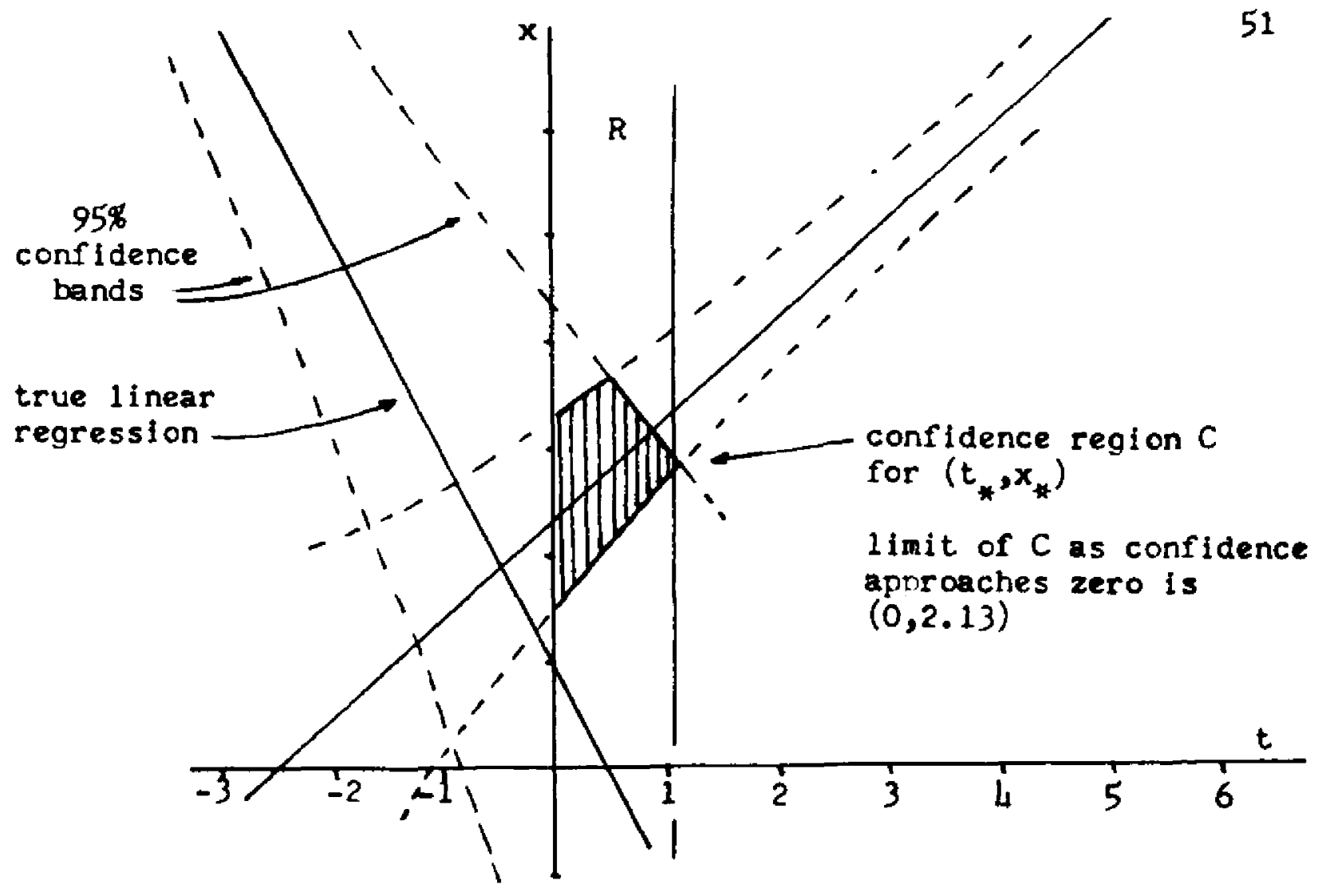

Figure 7

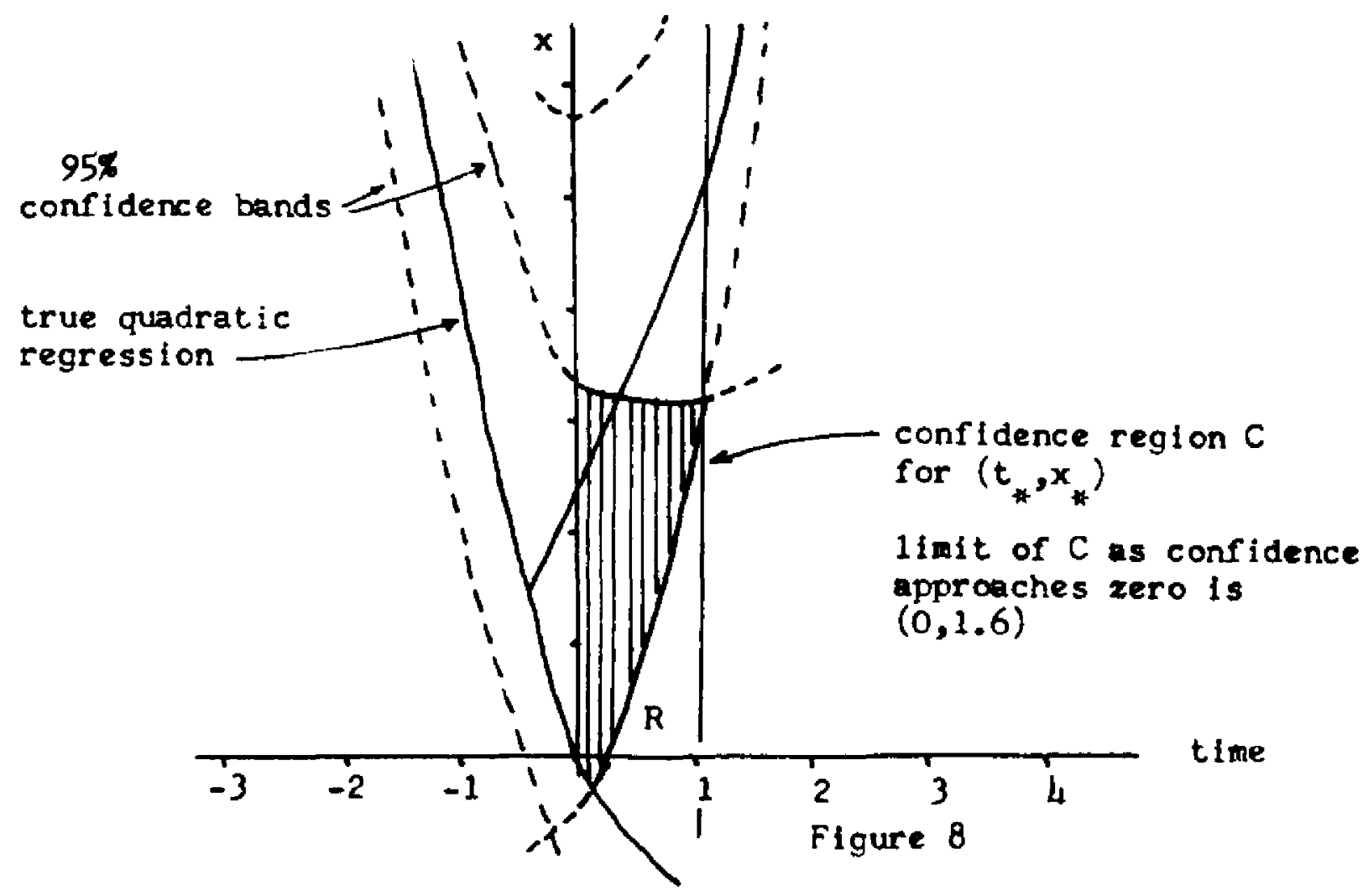


$2.6 \mathrm{H}_{1}$ and $\mathrm{n}_{2}$ Not Known

Suppose that $N_{1}$ and $N_{2}$ are not known, although $N=N_{1}+N_{2}$ is given. Then $L$ is a function not only of the continuous variables $\left(\alpha_{0}, \ldots, a_{r}, \beta_{0}, \ldots, \beta_{s}, \sigma_{1}{ }^{2}, \sigma_{2}{ }^{2}\right)$ but also of the single discrete variable $\mathrm{N}_{2}$. The number $\mathrm{N}_{2}$ is known if $\mathbf{N}$ and $\mathrm{N}_{1}$ are known. After obtaining the usual estimates for the continuous parameters our logic should be to further maximize $L$ by proper choice of $\mathrm{N}_{1}$ and $\mathrm{N}_{2}$. This can be done by examining the value of $L$ for all cholces of $N_{1}$ and $N_{2}$. This means that for every choice of $\left(N_{1}, N_{2}\right)$ a corresponding set of $\left(a_{0}, \ldots, b_{s}, \sigma_{1}{ }^{2}, \sigma_{2}{ }^{2}\right)$ nust be calculated and $L$ computed. The pair $\left(\mathrm{N}_{1}, \mathrm{H}_{2}\right)$ which maximizes $L$ is picked as the solution and the corresponding parameter estimates are used to estime $t_{*}$ as in previous sections.

Example. In the example of section 2.3 there were 21 sample pairs; we will now be working under the assumption that we don't know that $\left(\mathrm{N}_{1}-11, \mathrm{~N}_{2}=10\right)$ is the correct division between $X ' s$ and $Y$ 's. It is still assumed that $\sigma_{1}{ }^{2}=\sigma_{2}{ }^{2}$. The $\left(N_{1}, N_{2}\right)$ choices investigated were $(17,4),(16,5),(15,6), \ldots$, $(6,15),(5,16),(4,17)$. The smallest sample variance was, 8212 , corresponding to the choice $\left(N_{1}, N_{2}\right)=(11,10)$, which corresponds to the t-interval $[0,1]$. As seen before, the point estimate was $\hat{t}_{\#}=-0.49$ and the $95 \%$ improved confidence interval was $[0.0,0.07]$. Ordinarily it is not necessary to try all possible pairs, 
since by visual inspection of the graph of the observations one could delimit a reasonable range of pairs to try.

In making the cholce of $\left(N_{1}, N_{2}\right)$ based on maximum $L$ there is always a chance of error in the selection. The most pertinent questions concemhow good any procedure is in face of error. In problems of testing hypotheses the se questions are answered by calculating the power of a given test. In problems of interval estimation these questions can be answered as follows. If under assumptions $H$ we construct a confidence interval I having confidence $1-\alpha$, then under some alternative assumptions $A$ we must calculate an alternative confidence coefficient $1-\beta$ for the I determined by $\mathrm{H}$.

Now in our general problem with $\left(\mathbb{N}_{1}, N_{2}\right)$ known, the solution for estimating $t_{*}$ is to form the intersection $G$ of the $100 \%$ confidence interval $I:\left[s_{\mathbb{N}_{1}}, t_{1}\right]$ with a (1-e)100\% confidence interval $J$ obtained from a sample statistic. But with $\left(N_{1}, N_{2}\right)$ not exactly known, and hence estimated by maxinizing $L$, the confidence for $I$, and hence for $G$, is reduced. Our next task is to discuss this reduction. This will be done by finding the probability that the likelthood computed under one cholce of $\left(\mathbb{N}_{1}, \mathrm{~N}_{2}\right)$ exceeds the likelihood calculated under another choice of $\left(N_{1}, N_{2}\right)$. Consider first the two sample problem without regression assumptions.

\subsubsection{No Regression Assumptions}

Let $\left(x_{1}, \ldots, x_{N_{1}}\right)$ be an independent sample on a normal random randow variable $X$ with unknown mean $\mu_{1}$ and unknown variance $\sigma^{2}$. 
Let $\left(y_{1}, \ldots, y_{\mathbb{N}_{2}}\right)$ be an independent sample on a normal random variable $Y$ with unknown mean $\mu_{2}$ and the same unknown variance $\sigma^{2}$. Suppose these observations have occured at ordered times $\left(t_{1}, \ldots, t_{\mathbf{N}_{1}}\right.$, $\left.t_{N_{1}+1}, \ldots, t_{N_{1}+N_{2}}\right)$. The $t$ 's are not needed in the calculations but we wish to establish a regression point of view since we are searching for a time interval in which a change of regression takes place.

The likelihood function of the sample is

$$
\begin{gathered}
L\left(\mu_{1}, \mu_{2}, \sigma^{2}, N_{1}\right)=\prod_{i=1}^{N_{1}} \frac{1}{\sqrt{2 \pi} \sigma} e^{-\frac{1}{2 \sigma^{2}}\left[x_{1}-\mu_{1}\right]^{2}} . \\
\prod_{1=1}^{N} \frac{1}{\sqrt{2 \pi} \sigma} e^{-\frac{1}{2 \sigma^{2}}\left[y_{1}-\mu_{2}\right]^{2}}
\end{gathered}
$$

Suppose the choice $\mathrm{N}_{1}=$ has produced $\max \mathrm{Im} L$. The estimates are

$$
\begin{aligned}
& \hat{w}_{1}=m \\
& \hat{w}_{2}=w-n \\
& \hat{\mu}_{1}=\bar{x}_{m}=\frac{1}{m} \sum_{\substack{i=1 \\
n}}^{m} x_{i} \\
& \hat{\mu}_{2}=\bar{y}_{n}=\frac{1}{n} \sum_{i=1} y_{i} \\
& \hat{\sigma}^{2}=\frac{1}{m+n}\left\{\sum_{i=1}^{m}\left[x_{i}-\bar{x}_{m}\right]^{2}+\sum_{i=1}^{n}\left[y_{1}-\bar{y}_{n}\right]^{2}\right\} .
\end{aligned}
$$


The value of the likelihood function is thus

$$
L=\left(\frac{1}{\sqrt{2 \pi} \hat{\sigma}}\right)^{m+n} e^{-\frac{1}{2}(m+n)^{2}}
$$

Fy the likelihood principle we would then assert that the most probable interval for the change of regression is I: $\left[t_{m}, t_{m+1}\right]$. Let us determine the probability of this decision, namely,

$$
\operatorname{P=Pr}\left(L_{\mathbf{m}}>\mathrm{L}_{\mathbf{m}}, \mid \text { true } \mathrm{N}_{1}=\mathbf{m}\right)
$$

where $L_{\text {m }}$ denotes the likelihood computed when $N_{1}=$ observations are treated as $x^{\prime} s$. Thus $L_{m}$ is the likelihood computed under sone other choice of $N_{1}$. Notice that if the true $N_{1}$ equals $m$, and if $N_{1} \neq m$ is used in $L_{m}$, then there is a small group of interior data points all on either side of $x_{m}$ which have been misclassified as $x^{\prime} s$ or $y^{\prime} s$. Denote these points by $z_{1}, \ldots, z_{3}$ On the slde away from the nisclassification all clastiflcations are correct. Thus whenever $N_{1}=m^{\prime} \neq$ is used, the sample is being treated as having one of two forms:

$$
\left(x_{1}, \ldots, x_{r}, z_{1}, \ldots, z_{s}, y_{1}, \ldots, y_{n}\right)
$$

where $r+s=m$ and the $z^{\prime} s$ are treated as $y^{\prime} s$, or as

$$
\left(x_{1}, \ldots, x_{m}, z_{1}, \ldots, z_{s}, y_{1}, \ldots, y_{r}\right)
$$

where $r+s=n$ and the $z^{\prime} s$ are treated as $x^{\prime} s$. At this stage we must establish a useful decomposition Identity. 
56

Let there be given $\left(x_{1}, \ldots, x_{r}, z_{1}, \ldots, z_{s}, y_{1}, \ldots, y_{n}\right)$. Define

$$
\begin{aligned}
& n=r+s \\
& \bar{x}_{r+k}=\frac{1}{r+k}\left[\sum_{i=1}^{r} x_{i}+\sum_{i=1}^{k} z_{i}\right], k=0,1, \ldots, s \\
& \bar{z}_{k}=\frac{1}{k} \sum_{i=1}^{k} z_{1} \quad, k=0,1, \ldots, s \\
& \dot{y}_{k+n}=\frac{1}{k+n}\left[\sum_{i=5-k}^{s} z_{i}+\sum_{i=1}^{n} y_{i}\right], \quad k=0,1, \ldots, s \\
& s_{1}{ }^{2}=\sum_{r}^{r}\left[x_{i}-\bar{x}_{r+s}\right]^{2}+\sum_{s}^{s}\left[z_{i}-\bar{x}_{r+s}\right]^{2}+\sum_{n}^{n}\left[y_{i}-\bar{y}_{n}\right]^{2} \\
& s_{2}{ }^{2}-\sum^{r}\left[x_{1}-\bar{x}_{r}\right]^{2}+\sum_{s}^{s}\left[z_{1}-\bar{y}_{s+n}\right]^{2}+\sum^{n}\left[y_{1}-\bar{y}_{s+n}\right]^{2} \text {. }
\end{aligned}
$$

Now

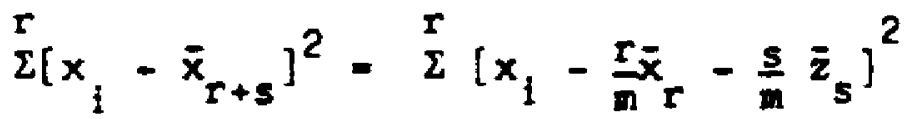

$$
\begin{aligned}
& =\stackrel{r}{\Sigma}\left[x_{i}-\frac{r+s}{m} \bar{x}_{r}+\frac{s}{m}\left(\bar{x}_{r}-\bar{z}_{s}\right)\right]^{2} \\
& \text { - } \stackrel{r}{\Sigma}\left[\left(x_{1}-\bar{x}_{r}\right)+\frac{s}{m}\left(\bar{x}_{r}-\bar{z}_{s}\right)\right]^{2} \\
& =\frac{r}{\Sigma}\left(x_{i}-\bar{x}_{r}\right)^{2}+\frac{r s^{2}}{m^{2}}\left(\bar{x}_{r}-\bar{z}_{s}\right)^{2}
\end{aligned}
$$

Similarly

$$
\stackrel{s}{\sum}\left[z_{1}-\bar{x}_{r+s}\right]^{2}=\sum_{\Sigma}^{s}\left(z_{1}-\bar{z}_{s}\right)^{2}+\frac{s r^{2}}{m^{2}}\left(\bar{z}_{s}-\bar{x}_{r}\right)^{2}
$$




$$
\begin{aligned}
& \stackrel{s}{\Sigma}\left[z_{1}-\bar{y}_{s+n}\right]^{2}=s\left(z_{1}-\bar{z}_{s}\right)^{2}+\frac{s n^{2}}{(s+n)^{2}}\left(\bar{z}_{s}-\bar{y}_{n}\right)^{2} \\
& n\left[y_{1}-\bar{y}_{s+n}\right]^{2}=\sum\left(y_{1}-\bar{y}_{n}\right)^{2}+\frac{n s^{2}}{(n+s)^{2}}\left(\bar{y}_{n}-\bar{z}_{s}\right)^{2} .
\end{aligned}
$$

Making these substitutions and adding proves the following lema.

Lemma.

$$
\begin{aligned}
& s_{1}{ }^{2}=\stackrel{r}{\Sigma}\left(x_{1}-\bar{x}_{r}\right)^{2}+\sum\left(z_{i}-\bar{z}_{s}\right)^{2}+\sum\left(y_{i}-\bar{y}_{n}\right)^{2}+\frac{r s}{r+s}\left(\bar{x}_{r}-\bar{z}_{s}\right)^{2} \\
& s_{2}^{2}=\stackrel{r}{\Sigma}\left(x_{1}-\bar{x}_{r}\right)^{2}+\Sigma\left(z_{i}-\bar{z}_{s}\right)^{2}+\sum\left(y_{i}-\bar{y}_{n}\right)^{2}+\frac{n s}{n+s}\left(\bar{y}_{n}-\bar{z}_{s}\right)^{2} .
\end{aligned}
$$

It will soon be convenient to refer to the first three terns of $s_{1}^{2}$ and $s_{2}^{2}$ as $C$.

Now consider the first form of the sample, thus assuming that the entire right side (y's) is always correctly classifled. Let $L_{a-s}$ be the likelihood obtained then $s z^{\prime}$ s are treated as $y^{\prime}$ '. The estimates in this case are

$$
\begin{aligned}
& \hat{\hat{\mu}}_{1}=\bar{x}_{r}=\frac{1}{r} \sum_{i=1}^{r} x_{i} \\
& \hat{\hat{\mu}}_{2}=\bar{y}_{n+s}=\frac{1}{n+s}\left[\sum_{i=1}^{s} z_{i}+\sum_{1=1}^{n} y_{i}\right] \\
& \hat{\tilde{\sigma}}^{2}=\frac{1}{r+s+n}\left[\sum_{i=1}^{s}\left(x_{i}-\bar{x}_{r}\right)^{2}+\sum_{i=1}^{s}\left(z_{i}-\bar{y}_{n+s}\right)^{2}+\sum_{i=1}^{n}\left(y_{i}-\dot{y}_{n+s}\right)^{2}\right]
\end{aligned}
$$


The value of the likelihood is

$$
L_{m-s} \cdot\left[\frac{1}{\sqrt{2 \pi} \hat{\hat{\sigma}}}\right]^{r+s+n} e^{-\frac{1}{2}(r+s+n)^{2}}
$$

The probability of the decision criterion is

$$
\begin{aligned}
\operatorname{P} & =\operatorname{Pr}\left(L_{m}>L_{m-s} \mid \operatorname{true} N_{1}=m\right) \\
& =\operatorname{Pr}\left(\hat{\sigma}^{2}>\hat{\sigma}^{2} \mid \operatorname{true} N_{1}=m\right) \\
& =\operatorname{Pr}\left(s_{2}^{2}>s_{1}^{2} \mid \operatorname{true} N_{1}=m\right) \\
& =\operatorname{Pr}\left(-1<\frac{s_{1}}{S_{2}}<1 \mid N_{1}=m\right) \\
& =\operatorname{Pr}\left(-1<\frac{\frac{r s}{r+s}\left(\bar{x}_{r}-\bar{z}_{s}\right)}{\frac{n s}{n+s}\left(\bar{y}_{n}-\bar{z}_{s}\right)}<1 \mid N_{1}=n\right)
\end{aligned}
$$

The last equality is obtained by using the decomposition lema.

In $P$ the numerator is normally distributed with

$$
\begin{aligned}
& \text { mean }-0 \\
& \text { variance }=\frac{r s}{r+s}\left(\frac{\sigma^{2}}{r}+\frac{\sigma^{2}}{s}\right)=\sigma^{2} .
\end{aligned}
$$

The denominator is normally distributed with

$$
\begin{aligned}
& \operatorname{mean}=\sqrt{\frac{n s}{n+s}}\left(\mu_{2}-\mu_{1}\right) \\
& \text { variance }-\frac{n s}{n+s}\left(\frac{\sigma^{2}}{n}+\frac{\sigma^{2}}{s}\right)=\sigma^{2}
\end{aligned}
$$


The covariance between numerator and denoninator is

$$
\begin{aligned}
\text { covariance } & =\sqrt{\frac{r s}{r+s} \sqrt{\frac{n s}{n+s}}} \operatorname{cov}\left(\bar{x}_{r}-\bar{z}_{s}, \bar{y}_{n}-\bar{z}_{s}\right) \\
& =\sqrt{\frac{r s}{r+s} \cdot \frac{n s}{n+s}} \operatorname{cov}\left(\bar{z}_{s}, \bar{z}_{s}\right) \\
& =\sqrt{\frac{r}{r+s} \cdot \frac{n}{n+s}} \sigma^{2} .
\end{aligned}
$$

Dividing the numerator and denominator of $P$ by $\sigma$ gives the form

$$
P=\operatorname{Pr}\left(-1<\frac{w_{1}}{w_{2}}<1 \mid N_{1}=m\right),
$$

where $w_{1}$ and $w_{2}$ are bivariate normal (bvn) components with means 0 and $\sqrt{\frac{n s}{n+s}}\left(\mu_{2}-\mu_{1}\right)$, variances 1 and 1 , and covariance $\sqrt{\frac{r}{r+s} \cdot \frac{n}{n+s}} \cdot \sqrt{n+s}^{\text {Thus }}$

$$
P=\int_{-1<\frac{w_{1}}{w_{2}}<1}\left(\text { bun density) dw } d w_{2} .\right.
$$

The region of integration is shaded below. All drawings will be made under the assumption $\mu_{2}>\mu_{1}$. If $\mu_{1} \leqq \mu_{2}$ the mathematical equations are stil1 correct, independent of the drawings.

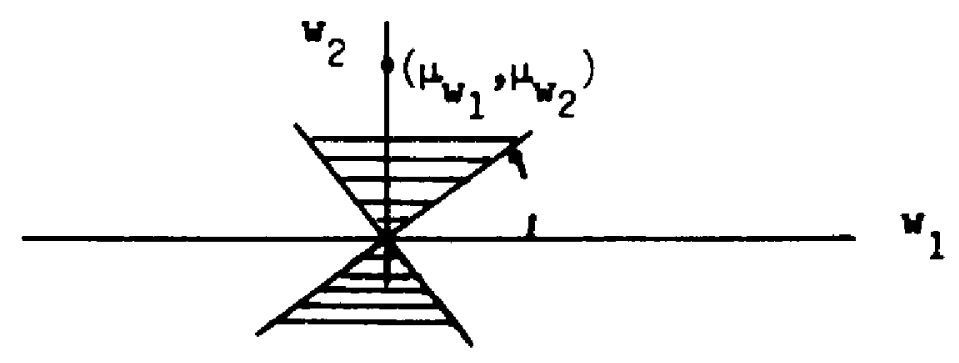


60

By the transformation

$$
\begin{aligned}
& u_{1}=\left(w_{1}-w_{w_{1}}\right) \cos \phi+\left(w_{2}-\mu_{w_{2}}\right) \sin \phi \\
& u_{2}=-\left(w_{1}-\mu_{w_{1}}\right) \sin \phi+\left(w_{2}-\mu_{w_{2}}\right) \cos \phi \\
& w_{1}=\mu_{w_{1}}+u_{1} \cos \phi-u_{2} \sin \phi \\
& w_{2}=\mu_{w_{2}}+u_{2} \sin \phi+u_{2} \cos \phi \\
& \tan 2 \phi=\frac{2 \sigma_{w_{1} w_{2}}}{\sigma_{w_{2}}-\sigma_{w_{1}}^{2}}
\end{aligned}
$$

we can obtain uncorrelated and hence independent normal random variables $u_{1}$ and $u_{2}$ (Cranmer, Mathematical Methods in Statistics, p. 279 .) Applying this result with $6=45$ degrees gives means zero and variances

$$
\begin{aligned}
& \sigma_{u_{1}}^{2}=1+\sqrt{\frac{n}{n+s} \cdot \frac{r}{r+s}} \\
& \sigma_{u_{2}}^{2}=1-\sqrt{\frac{n}{n+s} \cdot \frac{r}{r+s}}
\end{aligned}
$$

We transform further to independent normal $(0,1)$ variates by

$$
\begin{aligned}
& v_{1}=\frac{u_{1}}{\sigma_{u_{1}}} \\
& v_{2}=\frac{u_{2}}{\sigma_{u_{2}}}
\end{aligned}
$$


The point $(0,0)$ in $w$ coordinates has been transformed into the point

$$
(a, b)=\left(-\frac{\sqrt{\frac{n s}{n+s}} \frac{\mu_{2}-\mu_{1}}{\sqrt{2}}}{\sqrt{1+\sqrt{\frac{n}{n+s} \cdot \frac{r}{r+5}}}},-\frac{\sqrt{\frac{n s}{n+s} \frac{\mu_{2}-\mu_{1}}{\sqrt{2}}}}{\sqrt{1-\sqrt{\frac{n}{n+s} \cdot \frac{r}{r+s}}}}\right) .
$$

The new region of integration is shaded below:

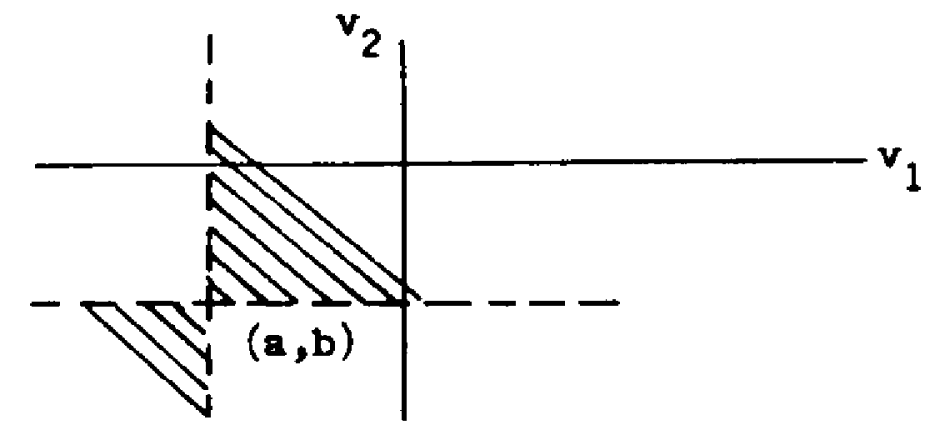

Finaliy,

$$
P=\int_{-\infty}^{a} n(0,1) d v_{1} \int_{-\infty}^{b} n(0,1) d v_{2}+\int_{a}^{\infty} n(0,1) d v_{1} \int_{b}^{\infty} n(0,1) d v_{2} .
$$

Consider next the second form of the sample, in which the left side ( $x^{\prime} s$ ) are correctly classified. Let $L_{m+s}$ be the likelihood obtained when $s z^{\prime} s$ are treated as $x$ 's. The estimates in this $c$ ase are

$$
\begin{aligned}
& \hat{\hat{\hat{\mu}}}_{1}=\bar{x}_{m+s}=\frac{1}{m+s}\left[\sum_{i=1}^{m} x_{i}+\sum_{i=1}^{s} z_{i}\right] \\
& \hat{\hat{\mu}}_{2}=\bar{y}_{r}-\frac{1}{r} \sum_{i=1}^{s} y_{1}
\end{aligned}
$$




$$
\hat{\boldsymbol{z}}_{2}=\frac{1}{r+s+n}\left[\sum_{i=1}^{\mathrm{n}}\left(x_{i}-\bar{x}_{m+s}\right)^{2}+\sum_{i=1}^{s}\left(z_{i}-\bar{x}_{m+s}\right)^{2}+\sum_{i=1}^{r}\left(y_{i}-\bar{y}_{r}\right)^{2}\right]
$$

The value of the likelihood is

$$
L_{m+s}=\left(\frac{1}{\sqrt{2 \pi}}\right)^{m+r+s} e^{-\frac{1}{2}(m+r+s)^{2}}
$$

The probability of the decision criterion is

$$
\begin{aligned}
P & =\operatorname{Pr}\left(L_{m}>L_{m+5} \mid N_{1}=m\right) \\
& =\operatorname{Pr}\left(\begin{array}{l|l}
\hat{\alpha}^{2}>\hat{\sigma}^{2} & N_{1}=n
\end{array}\right) .
\end{aligned}
$$

In this case the decomposition leme is applied to obtain

$$
\begin{aligned}
(r+s+n) \hat{\hat{\sigma}}^{2}-\sum_{i=1}^{m}\left(x_{i}-\bar{x}_{m}\right)^{2} & +\sum_{i=1}^{s}\left(z_{i}-\bar{z}_{s}\right)^{2}+\sum_{i=1}^{r}\left(y_{i}-\bar{y}_{r}\right)^{2} \\
& +\frac{m s}{m+s}\left(\bar{x}_{m}-\bar{z}_{s}\right)^{2}
\end{aligned}
$$

Then

$$
\begin{aligned}
P & \left.=\operatorname{Pr}\left|\frac{n s}{m+s}\left(\bar{x}_{m}-\bar{z}_{s}\right)^{2}>\frac{r s}{r_{s}}\left(\bar{y}_{r}-\bar{z}_{s}\right)^{2}\right| N_{1}=a\right) \\
& \left.=\operatorname{Pr}\left|-1<\frac{\sqrt{\frac{r s}{r+s}\left(\bar{y}_{r}-\bar{z}_{s}\right)}}{\sqrt{\frac{1 s}{m+s}}\left(\bar{x}_{m}-\bar{z}_{s}\right)}\right| N_{1}=n\right) .
\end{aligned}
$$


In this case the nunerator is normally distributed with

$$
\begin{aligned}
& \text { mean }=0 \\
& \text { variance }=\sigma^{2} .
\end{aligned}
$$

The denominator is normally distributed with

$$
\begin{aligned}
& \text { mean }=-\sqrt{\frac{m s}{m+s}}\left(\mu_{2}-\mu_{1}\right) \\
& \text { variance }=\sigma^{2}
\end{aligned}
$$

The covariance is

$$
\text { covariance }=\sqrt{\frac{r}{r+s} \cdot \frac{m}{m+s}} \sigma^{2} \text {. }
$$

Letting $w_{1}$ and $w_{2}$ be bivariate normal components with the parameters given above, we see that

$$
\begin{aligned}
P & =\operatorname{Pr}\left(-1<\frac{w_{1}}{w_{2}}<1 \mid N_{1}=m\right) \\
& =\int_{-1<\frac{w_{1}}{w_{2}}<1} \text { (bvn density)d } w_{1} d w_{2} .
\end{aligned}
$$

The region of integration is shaded below:

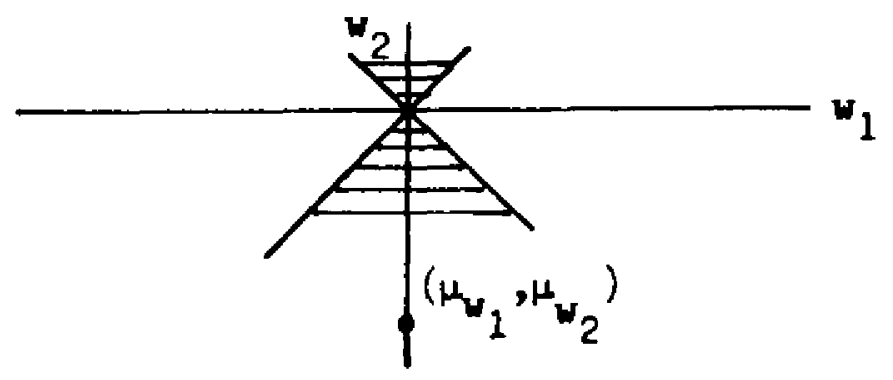


As before, we transform to uncorrelated variables and then to varlates with unit variance. The point $(0,0)$ in w-coordinates is transformed into v-coordinates of

$$
(a, b)=\frac{\sqrt{\frac{m s}{n+s}\left(\mu_{2}-\mu_{1}\right)} / \sqrt{2} \sqrt{\frac{m s}{n+s}\left(\mu_{2}-\mu_{1}\right) / \sqrt{2}}}{\sqrt{1+\sqrt{\frac{m}{m+s} \cdot \frac{n}{n+s}}}}, \sqrt{1-\sqrt{\frac{m}{n+s} \cdot \frac{n}{n+s}}}
$$

Then

$$
P=\int_{-\infty}^{a} n(0,1) d v_{1} \int_{-\infty}^{b} n(0,1) d v_{2}+\int_{a}^{\infty} n(0,1) d v_{1} \int_{b}^{\infty} n(0,1) d v_{2}
$$

These two results can be summarlzed in the following theorems.

Hypotheses. Let $\left(x_{1}, \ldots, x_{N_{1}}, y_{1}, \ldots, y_{N_{2}}\right)$ be independent observat lons on random variables $X$ and $Y$ having unknown means $\mu_{1}$ and $\mu_{2}$ and unknown but common variance $\sigma^{2}$. Supfose $N_{1}$ is unknown although $N=N_{1}+N_{2}$ is known. Let $L_{k}$ derote the value of the 11 kelihood function of the sample obtained by using the maximum likelihood estimates with $N_{1}=k$. Let the particular choice $N_{1}=\mathrm{m}$ and the associated $\hat{\mu}_{1}, \hat{\mu}_{2}, \hat{\sigma}^{2}$ produce a maximum 11 kelihood, $L_{m}$. Theorem. $\operatorname{Pr}\left(L_{m}>L_{m-s} \mid\right.$ true $\left.N_{1}-n\right)=p_{1} p_{2}+\left(1-p_{1}\right)\left(1-p_{2}\right)$ where

$$
p_{1}=\int_{-\infty}^{a} n(0,1) d v_{1} \text { with } a=\frac{\sqrt{\frac{n s}{n+s} \frac{\mu_{2}-\mu_{1}}{\sqrt{2}}}}{\sqrt{1+\sqrt{\frac{r}{r+s} \cdot \frac{n}{n+s}}}}
$$




$$
p_{2}=\int_{-\infty}^{b} n(0,1) d v_{2} \text { with } b=\frac{\sqrt{\frac{n s}{n+s} \frac{\mu_{2}}{n}-\mu_{1}}}{\sqrt{1-\sqrt{\frac{n}{n+s} \cdot \frac{r}{r+s}}}}
$$

Theoren. $\operatorname{Pr}\left(L_{m}>L_{m+s} \mid\right.$ true $\left.N_{1}=m\right)=p_{1} p_{2}+\left(1-p_{1}\right)\left(1-p_{2}\right)$ where

$$
\begin{aligned}
& p_{1}=\int_{-\infty}^{a} n(0,1) d v_{1} \quad \text { with } a=\frac{\sqrt{\frac{n s}{m+3}} \frac{\mu_{2}-\mu_{1}}{\sqrt{2}}}{\sqrt{1+\sqrt{\frac{m}{m+3} \cdot \frac{n}{n+s}}}} \\
& P_{2}=\int_{-\infty}^{b} n(0,1) d v_{2} \quad \text { with } b=\frac{\frac{\text { ms }}{m+s} \frac{\mu_{2}-\mu_{1}}{\sqrt{2}}}{\sqrt{1-\sqrt{\frac{m}{n+s} \cdot \frac{n}{n+s}}}}
\end{aligned}
$$

The choice $\mathrm{N}_{1}=m$ defines the interval $I:\left[t_{\mathrm{N}_{1}}, t_{\mathrm{N}_{1}+1}\right]$ most likely to contain the switch in regression from $\mu_{1}$ to $\mu_{2}$. The confidence for $I$, for fixed $s$, is defined as either of the probabilities given in the previous two theorems. The power for $I$, for fixed $s$, is now defined as $1 \mathrm{minus} e$ ither of the two previous probabilities when calculated under al ternative values for $N_{1}$. There are six cases to consider, and complete proofs will not be given because they are very similar to the proofs just presented. The variances and covariances remain the same. The rotation of 45 degrees remains the same. Only the means of the numerators and denominators change. Thus, explicit mention of these changes vill constituteour proof. 


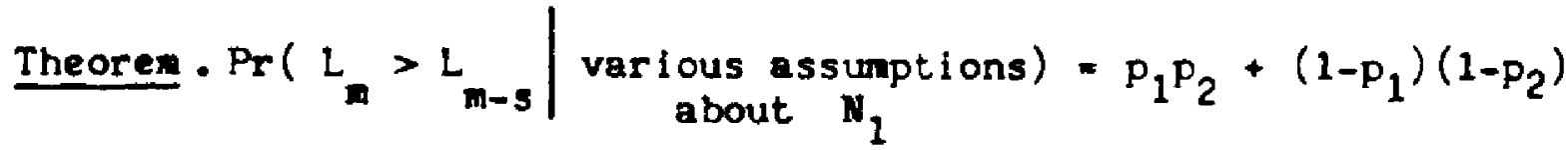

where $p_{1}=\int_{-\infty}^{a} n(0,1) d v_{1}$ and $p_{2}=\int_{-\infty}^{b} n(0,1) d v_{2}$

Let
$A=\frac{\frac{\mu_{2}-\mu_{1}}{\sqrt{2}}}{\sqrt{1+\sqrt{\frac{r}{r+s} \cdot \frac{n}{n+s}}}}$
$B=\frac{\frac{\mu_{2}-\mu_{1}}{\sqrt{2}}}{\sqrt{1-\sqrt{\frac{r}{r+3} \cdot \frac{n}{n+3}}}}$
$c=\frac{\frac{\mu_{2}-\mu_{1}}{\sqrt{2}}}{\sqrt{1+\sqrt{\frac{r}{r+s} \cdot \frac{m}{m+3}}}}$
$D=\frac{\frac{\mu_{2}-\mu_{1}}{\sqrt{2}}}{\sqrt{1-\sqrt{\frac{r}{r+3} \cdot \frac{n}{m+3}}}}$

Then

(1) For $N_{1}=\pi-s-t, s>0, t \geq 0$
$a=\frac{r-t}{r} \sqrt{\frac{r s}{r+s}} \cdot A$
$b=\frac{r-t}{r} \sqrt{\frac{r s}{r+s}} \cdot B$

(i1) For $w_{1}-w-s+t, s>0,0 \leq t \leq s$

$a=\left[\frac{s-t}{s} \sqrt{\frac{r s}{r+s}}-\frac{t}{s} \sqrt{\frac{n s}{n+s}} \cdot A \quad b-\left[-\frac{s-t}{s} \sqrt{\frac{r s}{r+s}}-\frac{t}{s} \sqrt{\frac{n s}{n+s}}\right] B\right.$

(iii) For $N_{1}=-s+t, \quad t \geq s>0$
$a=-\frac{n-t+s}{n} \sqrt{\frac{n s}{n+s}} \cdot A$
$b=-\frac{n-t+5}{n} \sqrt{\frac{n s}{n+3}} \cdot B$ 
Theorem. $\operatorname{Pr}\left(L_{m}>L_{m+s} \mid \underset{\text { about }}{\text { various as sumptions })=p_{1} p_{2}+\left(1-p_{1}\right)\left(1-p_{2}\right)}\right.$ about $N_{1}$

where $p_{1}, P_{2}, A, B, C, D$ are as previously defined. Then

(iv) For $w_{1}=m+s-t, s>0, s \geq t \geq 0$

$a=\left[-\frac{s-t}{s} \sqrt{\frac{r s}{r+s}}+\frac{t}{s} \sqrt{\frac{m s}{m+s}}\right] \cdot c \quad b=\left[\frac{s-t}{s} \sqrt{\frac{r s}{r+s}}+\frac{t}{s} \sqrt{\frac{m s}{m+s}}\right] \cdot D$

(v) For $\mathrm{N}_{1}=m+s-t, t \geq s>0$

$a=\frac{m-(t-s)}{m} \sqrt{\frac{m s}{m+s}} \cdot c \quad b=\frac{m-(t-s)}{m} \sqrt{\frac{m s}{m+s}} \cdot D$

(vi) For $N_{1}=m+s+t, t>0, s>0$
$a=-\frac{r-t}{r} \sqrt{\frac{r s}{r+s}} \cdot c$
$b=\frac{r-t}{r} \sqrt{\frac{r s}{r+s}} \cdot D$

Proof. We will first be concerned with probility statements of the form

$$
\begin{aligned}
\operatorname{P} & =\operatorname{Pr}\left(L_{n}>L_{n-s} J \operatorname{var} i \text { ous assumptions about } N_{1}\right) \\
& =\operatorname{Pr}\left(\begin{array}{c|c}
-1<\frac{\sqrt{r s}\left(\bar{x}_{r}-\bar{z}_{s}\right)}{\sqrt{\frac{n s}{n+s}}\left(\bar{y}_{n}-\bar{z}_{s}\right)}<1 & \begin{array}{c}
\text { various assumptions } \\
\text { about } N_{1}
\end{array}
\end{array}\right) .
\end{aligned}
$$

As noted before we need only calculate the means of the above numerator and denominator; in fact, we will exhibit merely

$$
\mu_{N}=E\left[\bar{x}_{r}-\bar{z}_{s}\right] \text { and } \mu_{D}=E\left[\bar{y}_{n}-\bar{z}_{s}\right] \text {. }
$$


(i)

$$
\begin{aligned}
\bar{x}_{r} & =\frac{1}{r}\left[\sum_{i=1}^{r-t} x_{i}+\sum_{i=r-t+1}^{r} x_{i}\right] \\
& =\frac{r-t}{r} \bar{x}_{r-t}+\frac{t}{r} \bar{x}_{t} \\
\mu_{N} & =\frac{r-t}{r} \mu_{1}+\frac{t}{r} \mu_{2}-\mu_{2}=-\frac{r-t}{r}\left(\mu_{2}-\mu_{1}\right) \\
\mu_{D} & =0
\end{aligned}
$$

(ii)

$$
\begin{aligned}
\bar{z}_{s} & =\frac{1}{s}\left[\sum_{i=1}^{t} z_{i}+\sum_{i=t+1}^{s} z_{i}\right] \\
& =\frac{t}{s} \bar{z}_{t}+\frac{s-t}{s} \bar{z}_{s-t}
\end{aligned}
$$$$
\mu_{N}=\mu_{1}-\frac{t}{s} \mu_{1}-\frac{s-t}{s} \mu_{2}=-\frac{s-t}{s}\left(\mu_{2}-\mu_{1}\right)
$$$$
\mu_{D}=\mu_{2}-\frac{t}{s} \mu_{1}-\frac{s-t}{s} \mu_{2}=\frac{t}{s}\left(\mu_{2}-\mu_{1}\right)
$$

(iii)

$$
\begin{aligned}
\bar{y}_{n} & =\frac{1}{n}\left[\sum_{i=1}^{t-s} y_{i}+\sum_{i=t-s+1}^{n} y_{i}\right] \\
& =\frac{t-s}{n} \bar{y}_{t-s}+\frac{n-t+s}{n} \bar{y}_{n-t+s}
\end{aligned}
$$




$$
\begin{aligned}
& \mu_{N}=0 \\
& \mu_{D}=\frac{t-s}{n} \mu_{1}+\frac{n-t+s}{n} \mu_{2}-\mu_{1}=\frac{n-t+s}{n}\left(\mu_{2}-\mu_{1}\right) .
\end{aligned}
$$

We will next be concerned with probability statements of the form

$$
\begin{aligned}
& P=\operatorname{Pr}\left(L_{m}>L_{m+s} \mid \text { various assuneptions about } N_{1}\right) \\
& -\operatorname{Pr}\left(-1<\frac{\sqrt{\frac{r s}{r+s}}\left(\bar{y}_{r}-\bar{z}_{s}\right)}{\sqrt{\frac{n s}{n+s}}\left(\bar{x}_{m}-\bar{z}_{s}\right)}<1 \mid \begin{array}{|}
\text { various assumptions } \\
\text { about } N_{1}
\end{array}\right) .
\end{aligned}
$$

We again demonstrate only $H_{N}$ and $\mu_{D}$.

(iv)

$$
\begin{aligned}
\bar{z}_{s} & =\frac{1}{s}\left[\sum_{i=1}^{s-t} z_{i}+\sum_{i=s-t+1}^{s} z_{i}\right] \\
& =\frac{s-t}{s} \bar{z}_{s-t}+\frac{t}{s} \bar{z}_{t} \\
\mu_{N} & =\mu_{2}-\frac{s-t}{s} \mu_{1}-\frac{t}{s} \mu_{2}=\frac{s-t}{s}\left(\mu_{2}-\mu_{1}\right) \\
\mu_{D} & =\mu_{1}-\frac{s-t}{s} \mu_{1}-\frac{t}{s} \mu_{2}=-\frac{t}{s}\left(\mu_{2}-\mu_{1}\right)
\end{aligned}
$$

(v)

$$
\begin{aligned}
\bar{x}_{x} & =\frac{1}{m}\left[\sum_{i=1}^{m-(t-s)} x_{1}+\sum_{i=m-(t-s)+1}^{m} x_{i}\right] \\
& =\frac{m-(t-s)}{m} \bar{x}_{m+s-t}+\frac{t-s}{m} \bar{x}_{t-s}
\end{aligned}
$$




$$
\begin{aligned}
& \mu_{N}=0 \\
& \mu_{D}=\frac{m-(t-s)}{m} \mu_{1}+\frac{t-s}{m} \mu_{2}-\mu_{2}=-\frac{m-(t-s)}{m}\left(\mu_{2}-\mu_{1}\right)
\end{aligned}
$$

$(v i)$

$$
\begin{aligned}
\vec{y}_{r} & =\frac{1}{r}\left[\sum_{i=1}^{t} y_{i}+\sum_{i=t+1}^{r} y_{i}\right] \\
& =\frac{t}{r} \bar{y}_{t}+\frac{r-t}{r} \bar{y}_{r-t}=\frac{r-t}{r}\left(\mu_{2}-\mu_{1}\right) \\
\mu_{N} & =\frac{t}{r} \mu_{1}+\frac{r-t}{r} \mu_{2}-\mu_{1}=0 \\
\mu_{D} & =0
\end{aligned}
$$

This completes the critical calculations for the proof.

All of the probabilities given in the previous theorems Involve the unknown quantity $\left(\mu_{2}-\mu_{1}\right)$, as they should. Various choices of this quantity can be made and plots of the various probablitties $P$ as a runction of $\left(\mu_{2}-\mu_{1}\right)$ could be constructed to aid in further understanding.

\subsubsection{With Regression Assumptions : Comnents}

If now regression assumptions are added the situation becomes very much more difficult. What is needed is a confidence for InJ. This can be written

$$
\text { Conf }[\text { InJ ] }=\operatorname{Conf}[J / I] \cdot \operatorname{Conf}[I] .
$$


The factor Conf [ I ] is associated with the probability $P$ of the decision which defines $I$,

$$
P=\operatorname{Pr}\left(L_{m}>L_{m !} \mid N_{1}=m\right) .
$$

After appropriate cancellations and reductions, and by analogy to the non regression case just discussed, $P$ becmes, for the first form of the sample,

$$
\begin{aligned}
P & =\operatorname{Pr}\left(s_{2}^{2}>s_{1}^{2} \mid N_{1}=n\right) \\
& =\operatorname{Pr}\left(\frac{s_{m}^{2}}{s_{m-s}^{2}}<1 \mid N_{1}=m\right)
\end{aligned}
$$

where

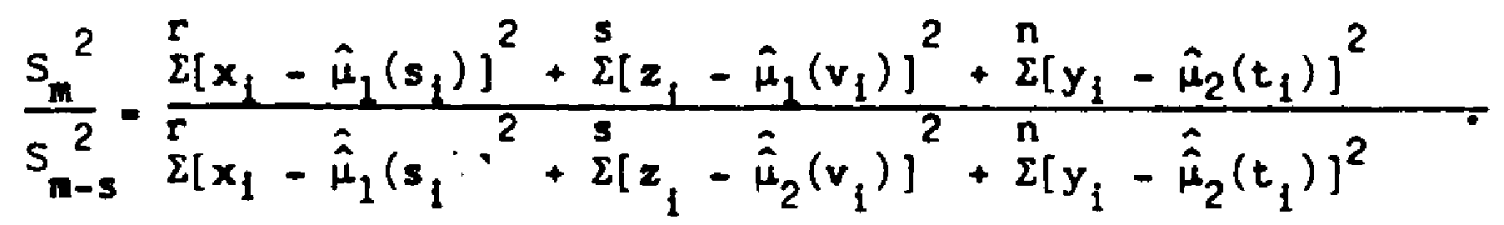

The variate $S_{m}^{2} / S_{m-s}^{2}$ is the ratio of two dependent non-central chi-square variates, and its distribution is not readily determined.

In the non regression case this difficulty was overcone by additively decomposing $S_{m}^{2}$ and $S_{m-s}^{2}$ before forming the ratio and cancelling certain cownon teras. But this procedure would seem to be impossible in the regression case since both the observations $x_{1}, z_{i}, y_{i}$ and the observable paraneters $s_{i}, v_{i}, t_{i}$ must be simultaneously decomposed. Typically, the difficulty arises with every $a_{i}$ from $\hat{\mu}_{1}$. Such an $a_{1}$ effectively contains the determinant of the matrix $M$ of squares and cross products of $s$ 's and $v$ 's In 1 ts denominator. This is because of the nature of $\mathrm{M}^{-1}$. 
But the determinant of $M$ contains s's and $V$ 's in a multiplicative way, and these two sets of variables cannot be separated in an additive manner. The problen is essentially that of an additive decomposition of, say, $(A+B)^{-1}$. It is doubtful whether any non additive decomposition, even if possible, would lead to manageable distribution results. Thus, chances for finding Conf [ I ] seem small.

The factor Conf [ $\mathrm{J} \mid \mathrm{I}]$ can be found by the methods of section 2.2.3 when $I$ is correctly defined. But if $I$ is incorrectly defined, and we want power-1ike statements for $J$, the non-decomposability probleas again confront us. The best we have been able to do is to give some qualitative approximations to the reduction in confidence.

First recall that Conf $[J \mid I$ ] requires calculations based on the student variate

$$
t=\frac{\frac{u}{\sigma_{u}}}{\sqrt{\frac{\Sigma\left[x_{i}-\hat{\mu}_{1}\left(s_{i}\right)\right]^{2}+\frac{s}{\Sigma}\left[z_{1}-\hat{\mu}_{1}\left(v_{1}\right)\right]^{2}+\sum^{n}\left[y_{1}-\hat{\mu}_{2}\left(t_{1}\right)\right]^{2}}{m+n-2 k-2}}}
$$

where $=r+s$

$$
u=\left(a_{0}+a_{1} t_{*}+\ldots+a_{k} t^{k}\right)-\left(b_{0}+b_{1} t_{*}+\ldots+b_{k} t_{*}^{k}\right) \text {. }
$$

Let $(H)$ refer to the situation that the $z^{\prime}$ 's are correctly classified as $x^{\prime} s$ in the first fam of the sample. Otherwise, (A), the $z$ 's have been isclassified and should have been treated as y's. 
73

We have just noted that it is difficult to additively pry apart the contribution of the $\left(v_{1}, z_{1}\right)$ to the determination of $\hat{p}_{1}$. Therefore we will consider an alternate student statistic which in no way involves the $\left(v_{1}, z_{1}\right)$.

Now under $A$ consider the numerator, $u$. In particular consider any $a_{1}$. From 1.5 with the notation of 2.2 .2

$$
a_{i}=\sum_{j=0}^{k} r^{i j} c_{j} \quad 1=0,1, \ldots, k
$$

where $\left(r^{i j}\right)=\left(r_{1 j}\right)^{-1}$

$$
\begin{gathered}
r_{i j}=\sum_{\varepsilon=1}^{r} s_{\varepsilon}{ }^{1} s_{\varepsilon}^{j}+\sum_{\varepsilon=1}^{s} v_{\varepsilon}{ }^{j} v_{\varepsilon}^{j}=r_{i j}^{(x)}+r_{i j}^{(z)} \\
c_{j}=\sum_{\varepsilon=1}^{r} x_{\varepsilon} s_{\varepsilon}^{j}+\sum_{\varepsilon=1}^{s} z_{\varepsilon} v_{\varepsilon}^{j}=c_{j}^{(x)}+c_{j}^{(z)} \\
j=0,1, \ldots, k
\end{gathered}
$$

Then $\quad E\left[a_{i}\right]=\sum_{j} r^{i j_{E}\left(c_{j}\right)}$

$$
\begin{aligned}
& =\sum_{j} r^{i j}\left(\sum_{a=1}^{r} s_{\alpha} j_{E\left[x_{a}\right]}+\sum_{\alpha=1}^{s} v_{a}^{j} j_{E\left[z_{\alpha}\right]}\right) \\
& =\sum_{j} r^{i j}\left(\sum_{\varepsilon=1}^{r} s_{\varepsilon}^{j} \sum_{p=0}^{k} a_{p} s_{\varepsilon}^{p}+\sum_{\varepsilon=1}^{s} v_{\varepsilon}^{j} \sum_{p=0}^{k} \beta_{p} v_{\varepsilon}^{p}\right)
\end{aligned}
$$




$$
\begin{aligned}
& =\sum_{j} r^{1 j}\left(\sum_{p=0}^{k} a_{p} r_{j p}^{(x)}+\sum_{p=0}^{k} \beta_{p} r_{j p}^{(z)}\right) \\
& =\sum_{j} r^{1 j} \sum_{p=0}^{k}\left[a_{p} r_{j p}^{(x)}+\beta_{p} r_{j p}^{(z)}\right] \\
\text { and } E[u] & =\sum_{i=0}^{k}\left[E\left(a_{i}\right)-\beta_{i}\right] t_{*}^{i}
\end{aligned}
$$

Since $x^{\prime} s$ and $z^{\prime}$ s have the same varlances, and since variance expressions are functionaliy independent of means, then the variance of $u$ is identical with the result obtained in 2.2 .3 :

$$
\sigma_{u}^{2}-\sigma^{2} \sum_{i=0}^{k} \sum_{j=0}^{k}\left(r^{1 j}+s^{i j}\right) t_{*}^{i+j}
$$

Consider now the alternative variate

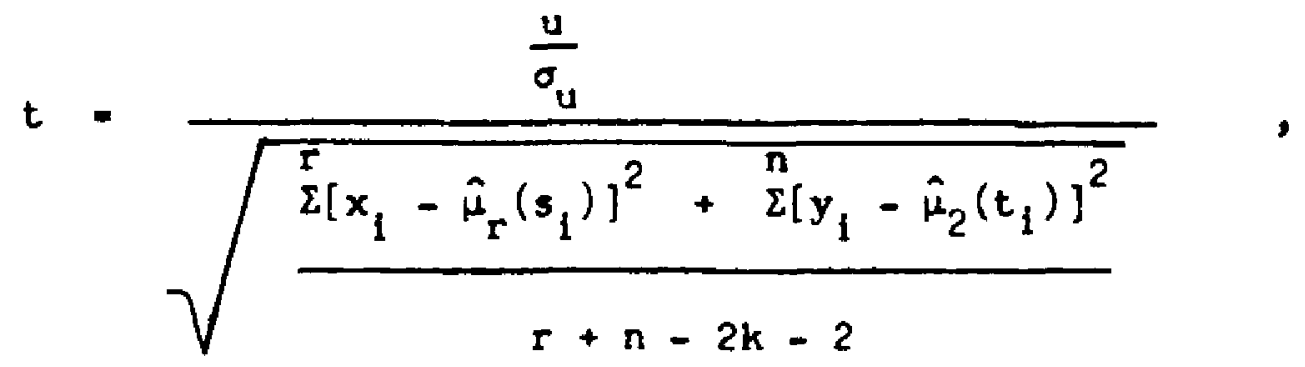

where $\hat{\mu}_{r}$ is calculated from only the $x$ 's and s's. The z's and $v$ 's are not being used. Under $H, t$ is a student variate with $(r+n-2 k-2)$ degrees of freedow. Under $A, t$ is non-central $t$ variate with $(r+n-2 k-2)$ degrees of freedion and 
parameter

$$
\delta=\frac{E[u]}{\sigma_{u}}
$$

Under $H$, fixed confidence intervals $I_{r}$ for given $1-\varepsilon$ can readily be constructed. Under $A$, tables of non-central $t$ can be consulted for various $\delta$ to obtain an alternative confidence for the fixed interval $I_{r}$. It seems plausible that the reduction in confidence for I would be comparable to the reduction actually observed $f a I_{r}$. For large in and $n$ this reduction should be nearly neglible. 
CHAPTER III

THE INTERSECTION OF SIMPLE LINEAR REGRESSIONS

CONTINUOUS MODEL

\subsection{Definition of the Problem}

Let there be given two sample functions $y_{1}(t)$ and $y_{2}(t)$ from two intersecting processes $Y_{t}^{(1)}$ and $Y_{t}^{(2)}$. Assume

$$
\begin{aligned}
& y_{t}^{(1)}=m_{1}(t)+e_{t}^{(1)}=a_{0}+a_{1} t+e_{t}^{(1)}, t_{1} \leq t \leq t_{2} \\
& Y_{t}^{(2)}=m_{2}(t)+e_{t}^{(2)}=b_{0}+b_{1} t+e_{t}^{(2)}, t_{3} \leq t \leq t_{4},
\end{aligned}
$$

where $e_{t}^{(1)}$ and $e_{t}^{(2)}$ are independent processes with covariance functions $\sigma_{j k}^{(1)}$ and $\sigma_{j k}^{(2)}$, where $a_{0}, a_{1}, b_{0}, b_{1}$ are constants to be estimated, and where $t_{1}, t_{2}, t_{3}, t_{4}$ are known $t$ ines with $t_{2}<t_{3}$. Our problem is to estimate $t_{*}$, the absclssa of intersection of $m_{1}$ and $m_{2}$, and to provide some distribution theory for the estinator.

For the continuous model we select maximum likelihood estimation whenever possible, and otherwise least squares estimators. The choice is dictated by how much is known or unknown about the given processes. This in turn depends partly on the covariance structures $\sigma_{j k}^{(1)}$ and $\sigma_{j k}^{(2)}$. We consider explicitly the case when the $e_{t}^{(i)}$ have the structure of a Fundamental Random Process (FRP) (see Mann, 1953). 
It will be assuned that for practical calculations some theory or form of physical equipment is available to perform integrations when introduced.

\subsection{Fundamental Random Process}

Assume that the $Y_{t}^{(i)}$ are processes for which the $\varepsilon_{t}^{(i)}$ are FRP's . Let $\varepsilon_{t, \tau}^{(i)}=e_{t+\tau}^{(1)}-\varepsilon_{t}^{(i)}$. Then $\sigma_{\varepsilon_{t, \tau}(1)}=c_{1} \tau, 1=1,2$. According to Theorem 2.3 of Mann (1953) the constants $c_{1}$ can be estimated with arbitrarily high precision by

$$
\hat{c}_{i}=\frac{1}{N_{i}} \sum_{n=1}^{N_{i}}\left[y_{i}\left(n \cdot \Delta t_{n}\right)-y_{i}\left(\overline{n-1} \cdot \Delta t_{n}\right)\right]^{2}, i=1,2,
$$

where the intervals $\left[t_{1}, t_{2}\right]$ and $\left[t_{3}, t_{4}\right]$ have been divided into $N_{1}$ equal parts of length $\Delta t_{n}$. The expected value of $\hat{c}_{1}$ is asymptotically $c_{i}$, and $\hat{c}_{i}$ converges in the mean to $c_{i}$. The variable $\left(\hat{c}_{1}-c_{i}\right)$ is stochastically of the order $x_{1}^{-1 / 2}$. The arbitrarily high precision is obtained by taking each $N_{i}$ as large as possible. Thus from the statistical estimation point of view we may regard the $c_{1}$ as known.

Consider now maximum likellhood estimation of the $a_{1}$. The estimation for the $b_{1}$ will be identical except for change in the time Interval of definition for the process. We thus suppress the superscript (i) distinction. For a subdivision $S_{n}:\left[t_{1}=s_{0}, s_{1}\right.$, $\left.s_{2}, \ldots, s_{n}=t_{2}\right]$ we assune, because we are dealling with an FRP, that the $\mathrm{Y}_{s_{j}}-\mathrm{Y}_{s_{j-1}}$ are independent1y and normally distributed 
with mean $\left(s_{j}\right)-m\left(s_{j-1}\right)$ and variance $c \cdot\left(s_{j}-s_{j-1}\right)$. The likelihood function is

$$
\begin{aligned}
L\left(S_{n} ; a_{0}, a_{1}\right)= & \prod_{j=0}^{n} \frac{1}{\sqrt{2 \pi c\left(s_{j}-s_{j-1}\right)}} . \\
& -\frac{\left[\left(Y_{\left.\left.s_{j}-Y_{s_{j-1}}\right)-\left(a_{0}-a_{0}\right)-a_{1}\left(s_{j}-s_{j-1}\right)\right]^{2}}^{2 c\left(s_{j}-s_{j-1}\right)}\right.\right.}{-} .
\end{aligned}
$$

Notice that $a_{0}$ vanishes by subtraction, and that the 11 kelihood is thus a function of the single parameter $a_{1}$ - By maximizing the logaritim of $L$ we obtain the single equation

$$
\left[\sum_{j=1}^{n}\left(Y_{s_{j}}-Y_{s_{j-1}}\right)-a_{1}\left(s_{j}-s_{j-1}\right)\right]\left[\frac{s_{j}-s_{j-1}}{s_{j}-s_{j-1}}\right]=0 .
$$

Since $Y_{t}$ is a process of second order we may pass to the stochastic linit in the mean to obtain

$$
\int_{t_{1}}^{t_{2}} d Y_{t}=a_{1} \int_{t_{1}}^{t_{2}} d t
$$

Hence

$$
\hat{a}_{1}=\frac{Y_{t_{2}}-Y_{t_{1}}}{t_{2}-t_{1}} .
$$

This estimate is normally distributed with zero mean and variance $c_{1} /\left(t_{2}-t_{1}\right)$ 
In practice one is given a single sample record $y(t)$, and the estimate is then

$$
\tilde{a}_{1}=\frac{y\left(t_{2}\right)-y\left(t_{1}\right)}{t_{2}-t_{1}} \text {. }
$$

This replacement can be $\operatorname{logically}$ justified. Introduce $I, \sum^{n}, f_{n}$, and the besic sample space $\Omega$ with elements $\omega$ by the extended identity

$$
I=\int_{t_{1}}^{t} d Y_{t}=1,1 . m \cdot \sum_{j=1}^{n}\left[Y_{t}{ }_{j}(\omega)-Y_{t}{ }_{j-1}(\omega)\right]-11 m \int_{n \rightarrow \infty} \int_{\Omega}^{n}(\omega) d P .
$$

This means

or that

$$
\lim _{n \rightarrow \infty} \int_{\Omega}\left[\frac{n}{\Sigma}\left(\omega_{n}\right)-I\right]^{2} d P=0
$$

$$
\lim _{n \rightarrow \infty} \int_{\Omega} f_{n}^{2}(\omega) d P=0 .
$$

From Hobson (Theory of Functions of a Real Variable, 2, Dover (1957), paragraphs 168-172) the existence of such limits implies that $f_{n}(w)$ converces to zero at almost every point in $\Omega$. That is to say, the ordinary Riemann integral of almost every sample function exists. Thus $\int_{1}^{t_{2}}$ exists for almost all $y(t)$ and equals $y\left(t_{2}\right)-y\left(t_{1}\right)$. The estimation of $b_{1}$ would procede in like fashion. Mann has shown that the estimates are unblased.

It is disappointing that the raximu likelihood procedure as formulated here, using the FRP assumptions that changes in $Y_{t}$ 
are independent and norme 1 , does not lead to direct estimation of ${ }^{a} 0^{\circ}$ If we attempt to formulate M.L. at the $Y_{t}$ level, matters become quite complicated for an FRP because we can no longer assume that consecutive observations are independent. Furthermore, assumptions would need now be made about the covarlance of $Y_{t}$, whereas before such assumptions had only to be made about changes in $Y_{t}$. Other writers have been able to avold this problen by assuming that at time $t_{1}$ the process is virtualiy constant and thus a can be estimated directly by

$$
\tilde{a}_{0}=y\left(t_{1}\right)
$$

Adopting this point of view we would set

$$
\tilde{a}_{0}=y\left(t_{1}\right) \text { and } \quad \tilde{b}_{0}=y_{2}\left(t_{4}\right) \text {. }
$$

Thus in our mode1, a particle starts out with virtually no variation and pursues a linear course in which its random deviations from the course get larger. Then at some unknown time, $t_{*}$, it is influenced to alter its direction and continue in a 11 near course, and does so unt 11 its randon variation bec mes neglible.

Finally, the estimate for $t_{*}$ is obtained by equating $m_{1}$ and $m_{2}$ :

$$
\begin{aligned}
\tilde{a}_{0}+\tilde{a}_{1} t_{*} & =\tilde{b}_{0}+\tilde{b}_{1} t_{*} \\
\tilde{t}_{*} & =\frac{\check{a}_{0}-\tilde{b}_{0}}{\tilde{b}_{1}-\tilde{a}_{1}}
\end{aligned}
$$

This random variable is the ratio of the constant $h=\tilde{a}_{0}-\tilde{b}_{0}$ and a 
normal variate $z=\tilde{b}_{1}-\tilde{a}_{1}$ having mean $\mu_{z}=b_{1}-a_{1}$ and known variance $\sigma_{z}{ }^{2}=\sigma_{b_{1}}^{2}+\sigma_{a_{1}}^{2}=c_{2} /\left(t_{4}-t_{3}\right)+c_{1} /\left(t_{2}-t_{1}\right)$. Three cases must be considered in stating the distribution of $t_{*}$.

Suppose first that $h>0$. Let $k$ be a fixed point on the distribution of $z$. Then

$$
\operatorname{Pr}\left[\frac{h}{z}<k\right]=\left\{\begin{array}{ll}
\frac{1}{2}+\int_{h / k}^{\infty} d N \quad \text { for } k>0 \\
\frac{1}{2} & \text { for } k=0 \\
\frac{1}{2}-\int_{-\infty}^{h / k} d N & \text { for } k<0
\end{array},\right.
$$

where $N$ denotes the distribution of $z$.

Secondly, if $h<0$ we rewrite $t_{*}$ as follows:

$$
\tilde{t}_{\hbar}=\frac{b_{0}-a_{0}}{\tilde{a}_{1}-\tilde{b}_{1}} \text {. }
$$

Aga in the numerator is constant, but this time the denominator is the normal varlate $z_{*}=\tilde{a}_{1}-\tilde{b}_{1}$. The distribution of $\tilde{t}_{*}$ is obtained from the previous result by replacing $d N$ by $d_{*}$, where $N_{*}$ is the distribution of $z_{*}$.

Thirdly, if $h=0$, the solution for $t_{*}$ is

$$
\tilde{t}_{*}=\frac{\tilde{b}_{1}}{\tilde{a}_{1}} \text {. }
$$

This time $\tilde{t}_{*}$ is a ratio of independent normal variates. 
Let

$$
\begin{aligned}
& A=\frac{E\left[\tilde{b}_{1}\right]}{E\left[\tilde{a}_{1}\right]}=\frac{b_{1}}{a_{1}} \\
& u=\tilde{b}_{1}-A \cdot \tilde{a}_{1} \text {. }
\end{aligned}
$$

Then $u$ is normally distributed with zero mean and variance

$$
\sigma_{u}^{2}=\sigma_{b_{1}}^{2}+A^{2} \sigma_{a_{1}}^{2}=\frac{c_{2}}{t_{4}-t_{3}}+\frac{b_{1}^{2}}{a_{1}^{2}} \cdot \frac{c_{1}}{t_{2}-t_{1}}
$$

Confidence linits for $A$ can be found from the distribution of $u$ in a standard manner.

\subsection{Other Investigations}

In 1951 Robblns and Monro presented a distribution-free stochastic method for solving the equation $f(x)=a$ for $x$. The function $f(x)$ is an unknown regression, and observations of random variables about $f(x)$ are avallable at any stage $x$. The constant a is known. The solution consists of constructing a sequence of approximations which converge in some probabilistic sense to the true value $x=\theta$. In 1952 Kieffer and Wolfowitz modified the RM procedure to estimate maxima of $f(x)$. In 1956 Derman summarlzed all work on RM in single paper.

If the objective in our problem of estimating intersections of regressions is to locate $a \max$ or a $\mathrm{m}$, then the results of kiefer and Wolfowitz nay solve the problem in the continuous case. There 


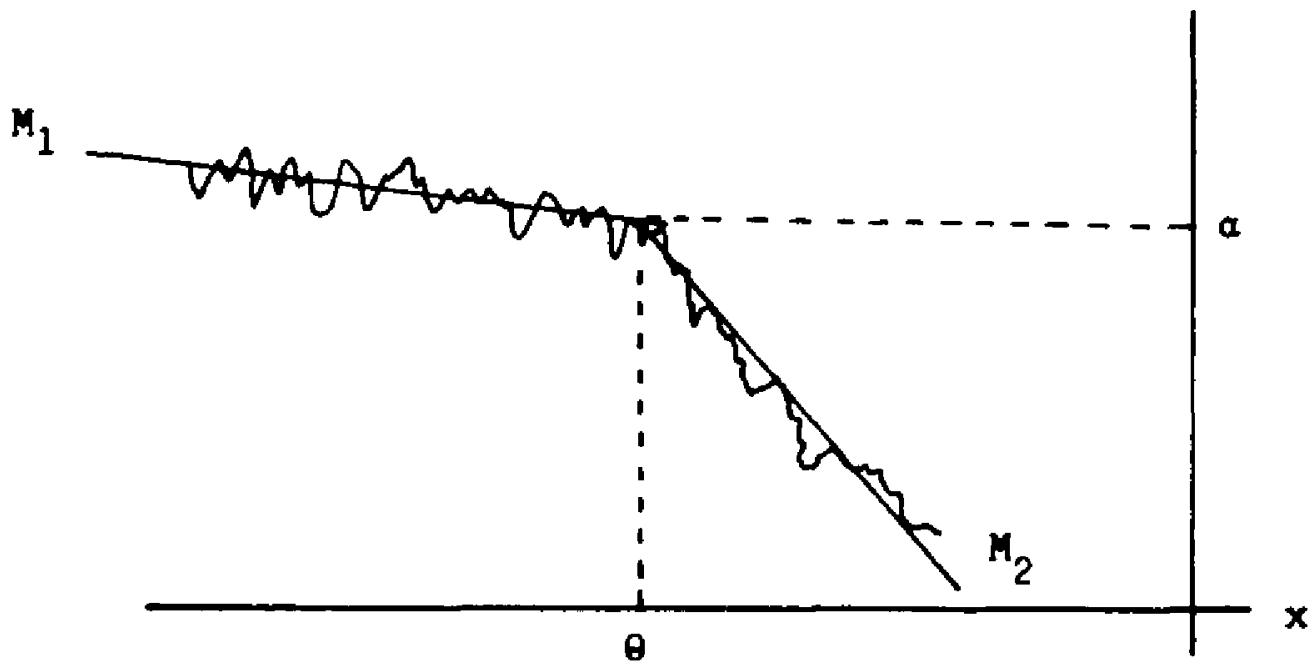

Figure 9

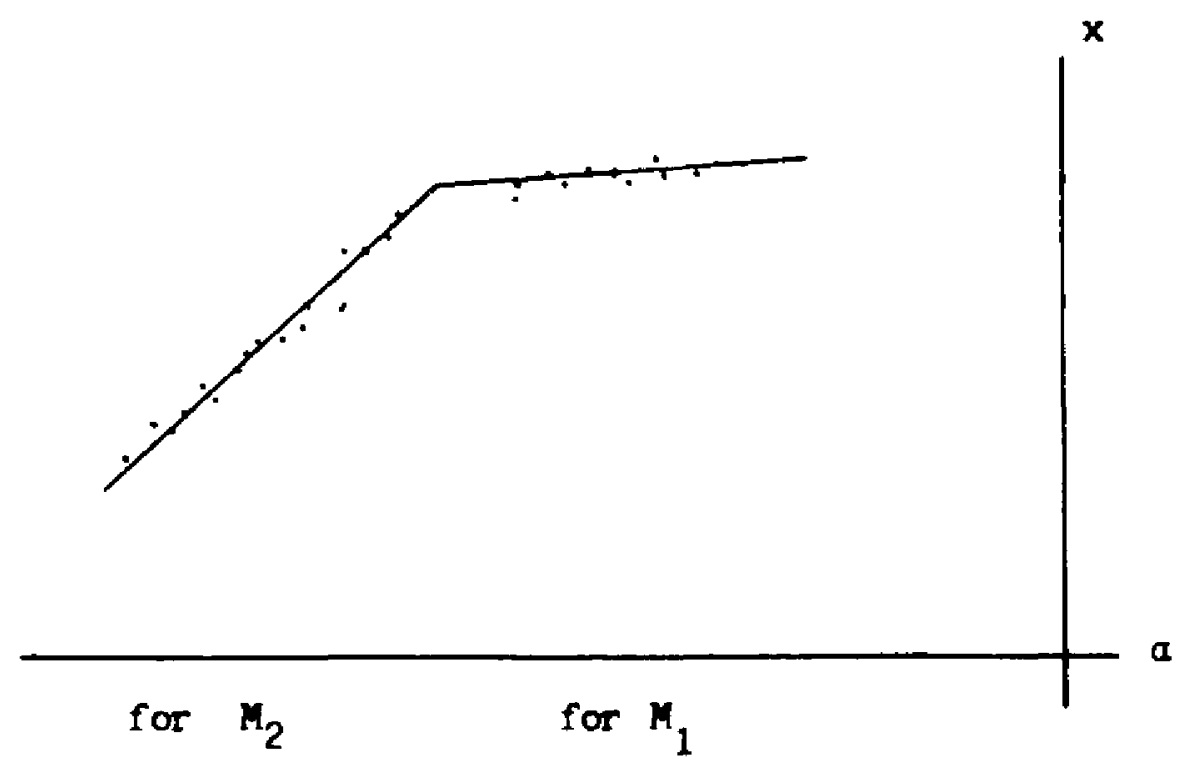

Figure 10 
could be practical difficulties if slow convergence is encountered. However, our interest in intersecting regressions may be to locate a change point $(\theta, a)$ of the form show in figure 9 . The functions $M_{1}$ and $M_{2}$ are branch regressions of $f(x)$. The $R M$ procedure could be used to approximate $(\theta, a)$ as follows. Relative to $M_{1}$ estimate several values $\theta_{i}$ corresponding to various given values of $a_{i}$. Do the same for $M_{2}$. Plot these results, as in figure 10 . Then estimate the point of intersection of best fit curves through the two clusters of points. If the clusters are wide spread, repeated estimates at any $a_{i}$ can be obtained by using a different starting point in the calculation. Multiple estimates at any $a_{1}$ can then be averaged and the spread of the elusters condensed. A thorough study of this approach could be the topic of another paper.

In 1959 E.J. Williams published his Regression Analysis . He does not consider problens of intersecting regressions, but some of the intermediate results of this thesis are similar in nature to Williams's work on zeros of polynomials.

In $195^{\circ}$ Box and Hunter determined confidence regions for solutions to simultaneous linear equtions. One ap-1ication of their results is the location of extrem.

In conclusion we note that Williams, Box and Hunter, and the author of this thesis have all encountered practical difficulties when sample is just bad enough to destroy the expected shapes of needed confidence regions. 
Anderson, T.W. (1958), An Introduction to Multivariate Statistical Analys is, Wiley, New York.

Box, G.E.P. and Hunter, J.S. (1954), A confidence region for the solutions of a set of simultaneous equations with an application to experinental desigh, Biometrika 4l, Parts 1 and 2, 190-196.

Craner, H. (1945), Mathematical Methods of Statistics, Princeton University Press, Princeton.

Daniels, H. (1954). A distribution-free test for regression parameters, Ann. Math. Stat. 25, 499-513.

Farrel1, R. (1962), Bounded length confidence Intervals for the zeros of a regression function, Ann. Math. Stat. 33, 237-247.

Fisher, R.A. (194l), Statistical Methods for Research Workers, 01 iver and Boyd, Edinburgh, 144-146.

Hobson, E.W. (1957), Theory of Functions of a Real Variable, 2, Dover, New York.

Kastenbaum, Marvin (1959), A confidence interval on the abscissa of the point of intersection of two fitted linear regressions, J. Amer. Stat. Assoc. 53, 873-880.

Kempthorne, O. (1952), Design and Amalys Is of Experiments, Wiley, New York.

Mann, H.B. (1949), Analys is and Design of Experiments, Dover, New York.

Mann, H.B. (1953), Introduction to the theory of stochastic processes depending on a continuous parameter, Hat lonal Bureau of Standards Applied Mathematics Serles 24, U.S. Government Printing Offlce, Washington.

Mann, H.B. (1954), A theory of estimation for the fundamental random process and the Ornstein-Uhienbeck process, Sankhya 13, $325-350$.

Mood, A.M. (1950), Introduction to Mathenatical Statistics, McGraw-H111, New York. 
Page, E. (1955), A test for a change in a parameter occuring at an unknown point, Biometrika 42, 523-527.

Page, E. (1957), On problems in chich a change in a parameter occurs at an unknown point, Blometrika $44,248-252$.

Patnaik, P. (1949), The non-central chi-square and F-distributions and their applications, Biometrika 36, 202-232.

Quandt, R. (1958), The estimation of the parameters of linear regression system obeying two separate reqlmes, J. Amer. Stat. Assoc. 53, 873-880.

Resnikoff, G. and Lieberman, G. (1957), Tables of the Non-central t-Distribution, Stanford University Press, Stanford.

Robbins, H. and Monro, S. (1951), A stochastic approximation method, Ann. Math. Stat. 22, 400-407.

Scheffe, H. (1959), The Analysis of Variance, Wile, New York.

Schever, E. (1960), Simultanious estimation for means or medians of dependent random variables without distribution assumptions, Ph.D. dissertation, University of Callfornia at Los Angeles.

Williams, E. (1959), Regression Analysis, Wiley, New York. 


\section{AUTOBIOGRAPHY}

I, Donald Edward Robison, was born in Alturas, California, July 1, 1931. I attended grade school and two years of high school in San Gabriel, California. I completed my high school education in Oregon where I graduated fron Springfleld High School in 1948. In 1952 I received the degree Bachelor of Science in Mathematics from the University of Oregon. From 1952 to 1954 I was in the United States Army. I was stationed at White Sands Proving Grounds in New Mexico, working under the military occupation speciality of Mathematics Research Assistant. During this time I began my graduate work as a special student at New Mexico A. and M. College. In 1954 I came to Ohio State University and in 1956 I was granted the degree Master of Science in Mathematics. Hy thesis was written in the field of mathematical statistics. The years 1956 to 1959 were spent at Ohio State University where I was preparing for the Ph.D. degree in Mathematics in the fleld of Mathenatical Statistics. During this time I held positions of graduate assistant, research assistant, research associate, and assistant instructor. In 1959, after passing the general examination for the Ph. D, degree, I jolned the technical staff of Space Technology Laboratories of Los Angeles as a Mathematical Statisticlan. Granted a leave of absence from that organlzation for $1961-1962$, I returned to Ohlo State University to complete my doctoral dissertation. 